Draft Version July 18, 2018

Typeset using LATEX twocolumn style in AASTeX61

\title{
X-RAY CENSUS OF MILLISECOND PULSARS IN THE GALACTIC FIELD
}

\author{
Jongsu Lee,${ }^{1}$ C. Y. Hui,${ }^{2}$ J. Takata,${ }^{3}$ A. K. H. Kong,${ }^{4}$ P. H. T. Tam,${ }^{5}$ and K. S. Cheng ${ }^{6}$
}

\footnotetext{
${ }^{1}$ Department of Astronomy, Space Science and Geology, Chungnam National University, Daejeon 34134, Korea

${ }^{2}$ Department of Astronomy and Space Science, Chungnam National University, Daejeon 34134, Korea

${ }^{3}$ Institute of Particle physics and Astronomy, Huazhong University of Science and Technology, China

${ }^{4}$ Institute of Astronomy, National Tsing Hua University, Hsinchu, Taiwan

${ }^{5}$ School of Physics and Astronomy, Sun Yat-sen University, Zhuhai 519082, China

${ }^{6}$ Department of Physics, University of Hong Kong, Pokfulam Road, Hong Kong
}

(Received July 18, 2018)

\section{ABSTRACT}

We have conducted a systematic survey for the X-ray properties of millisecond pulsars (MSPs). Currently, there are 47 MSPs with confirmed X-ray detections. We have also placed the upper limits for the X-ray emission from the other 36 MSPs by using the archival data. We have normalized their X-ray luminosities $L_{x}$ and their effective photon indices $\Gamma$ into a homogeneous data set, which enable us to carry out a detailed statistical analysis. Based on our censored sample, we report a relation of $L_{x} \simeq 10^{31.05}\left(\dot{E} / 10^{35}\right)^{1.31} \mathrm{erg} / \mathrm{s}(2-10 \mathrm{keV})$ for the MSPs. The inferred $\mathrm{X}$-ray conversion efficiency is found to be lower than previously reported estimate that could be affected by selection bias. $L_{x}$ also correlates/anti-correlates with the magnetic field strength at the light cylinder $B_{L C} /$ characteristic age $\tau$. On the other hand, there is no correlation between $L_{x}$ and their surface magnetic field strength $B_{s}$. We have further divided the sample into four classes: (i) black-widows, (ii) redbacks, (iii) isolated MSPs and (iv) other MSP binaries, and compare the properties among them. We noted that while the rotational parameters and the orbital periods of redbacks and black-widow are similar, $L_{x}$ of redbacks are significantly higher than those of black-widows in the $2-10 \mathrm{keV}$ band. Also the $\Gamma$ of redbacks are apparently smaller than those of black-widows, which indicates the $\mathrm{X}$-ray emission of redbacks are harder than that of black-widows. This can be explained by the different contribution of intrabinary shocks in the X-ray emission of these two classes.

Keywords: stars: binaries: general — pulsars: general — X-rays: general 


\section{INTRODUCTION}

Since the discovery of the first millisecond pulsar (MSP) PSR B1937+21 (Backer et al. 1982), a distinct class in the pulsar population, characterised by a rotational period $P \lesssim 20 \mathrm{~ms}$ and a spin-down rate $\dot{P} \lesssim 10^{-18} \mathrm{~s} \mathrm{~s}^{-1}$, has been established (Manchester et al. 2005). It is generally accepted that MSPs are formed when an old neutron star has been spun up through accreting mass and angular momentum from its companion (Alpar et al. 1982; Radhakrishnan \& Srinivasan 1982; Fabian et al. 1983).

In the recent years, the population of MSPs have been expanded significantly (e.g. Hui 2014 for a recent review). Multi-wavelength follow-up investigations of unidentified $\gamma$-ray objects detected by Fermi Gammaray Space Telescope have been demonstrated to be successful in discovering MSPs (Abdo et al. 2013; Hui et al. 2015; Ray et al. 2012). Currently, there are $~ 200$ MSPs found in the Galactic field (Manchester et al. 2005).

Apart from expanding the population, observations in the recent years have also shown that MSPs can be further divided into several sub-classes, including blackwidows (referred as BWs hereafter), redbacks (referred as RBs hereafter) as well as isolated MSPs.

BWs are binary MSPs bounded in tight orbits $\left(P_{b} \lesssim\right.$ $20 \mathrm{hrs}$ ) with companions of only a few percents of solar mass (cf. Hui 2014). A prototypical example of this class is PSR B1957+20 which is a binary system with a 9.2 hrs orbit containing a MSP with $P=1.6 \mathrm{~ms}$ and a companion of $M_{c} \sim 0.02 M_{\odot}$ (Fruchter et al. 1988). Another characteristics of the BWs is the presence of radio eclipse. In the case of PSR B1957+20, the eclipses of the radio pulsations occur regularly for $\sim 10 \%$ of its orbit (Fruchter et al. 1988).

RBs form a relatively new class of MSPs which has only emerged for a decade. Their orbital periods $P_{b}$ span a somewhat larger range than that of BWs (i.e. $P_{b} \lesssim 20 \mathrm{hrs}$ ) and their companions are generally nondegenerate and more massive $\left(M_{c} \sim 0.2-0.4 M_{\odot}\right)$. The most remarkable characteristic of $\mathrm{RBs}$ is that these systems can possibly swinging between rotationpowered state and accretion-powered state. ${ }^{1}$ The prototypical example of this class in the Galactic field is PSR J1023+0038 (Archibald et al. 2009,2010), which was firstly identified as a low-mass X-ray binary (Homer et al. 2006) and subsequently a radio MSP was discovered and a former accretion disk was found to disappear (Archibald et al. 2009). Interestingly, since 2013 late

\footnotetext{
${ }^{1}$ The first case of a swinging pulsar PSR J1824-2452I was found in globular cluster M28 (Papitto et al. 2013).
}

June, the radio pulsation of this system has disappeared and a new disk has been formed, which indicates the system has re-entered the accretion-powered state (Stappers et al. 2014; Takata et al. 2014; Li et al. 2014).

There are $\sim 30 \%$ of the known MSPs in the Galactic field are found to be isolated (Manchester et al. 2005). As MSPs are considered to be the off-springs from the evolution of compact binaries, the existence of isolated MSPs has raised a question for their origins. One possible explanation for their solitudes is that their companions have been evaporated in the presence of the high energy radiation and/or the relativistic wind particles from the companion MSPs (van den Heuvel \& van Paradijs 1988).

Statistical analyses of the X-ray properties of pulsars can place constraints on the radiation mechanisms (e.g. Seward \& Wang 1988; Becker \& Trümper 1997; Possenti et al. 2002; Kargaltsev \& Pavlov 2008; Vink et al. 2011; Prinz \& Becker 2015; Shibata et al. 2016). Most of these studies focused on investigating the empirical relation between the X-ray luminosities $L_{x}$ and the spin-down power $\dot{E}$. The reported relations are found to diverse in the literature. This can be ascribed to a number of factors. First, the choice of energy bands can affect the best-fit relation. For those have included the soft band $(\lesssim 2 \mathrm{keV}$ ) in their study (e.g. Prinz \& Becker 2015), there can be contribution from the neutron star cooling in the young/middle-aged pulsars which should not covary with $\dot{E}$. Moreover, soft X-ray fluxes are more sensitive to the uncertainties due to the interstellar absorption.

The diversity can also be related to the different sample selection criteria in different studies. Pulsar population is heterogeneous, which comprises many different subclasses: such as young pulsars (e.g. Crab), high magnetic-field pulsars, MSPs. The X-ray emission properties can be vary among different classes (e.g. Possenti et al. 2002; Shibata et al. 2016). Therefore, investigations that include all the X-ray detected pulsars in their samples are subjected to a large scattering. Therefore, recent statistical studies of pulsars are focused on particular sub-classes (e.g. Shibata et al. 2016). However, there is no corresponding updated analysis of MSPs.

A detailed statistical analysis of X-ray detected MSPs dated back to Possenti et al. (2002) which suggest a best-fit relation of $\log L_{x}=(1.38 \pm 0.10) \log \dot{E}-(16.36 \pm$ 3.64 ) in $2-10 \mathrm{keV}$ for ten MSPs with confirmed X-ray detections at that time (cf. Fig. 2 in Possenti et al. 2002).

With a significantly enlarged sample, it is timely to reexamine the X-ray emission properties of MSPs. Also, as 
different sub-classes of MSPs have now been identified, it is interesting to compare the physical and emission properties among different classes. In this paper, we present the results from a detailed statistical analysis of the X-ray properties of MSPs in the Galactic field. For the MSPs residing in globular clusters, as the formation processes are different from those in the Galactic field, their emission properties can possibly be different (Hui, Taam \& Cheng 2010). Hence the globular cluster MSPs are excluded in this study.

\section{DATA COLLECTION \& NORMALIZATION}

Using ATNF pulsar catalog of the version in April 2017 (Manchester et al. 2005), we firstly compiled a list of radio pulsars based on the following criteria:

\section{Rotational period $P<20 \mathrm{~ms}$}

\section{Excluding the pulsars in globular clusters}

There are 197 radio pulsars fulfilling the aforementioned criteria. We subsequently searched for the literature which is relevant to the X-ray properties of the pulsars in our list. Since different studies have adopted different energy ranges and different spectral models in their analyses, the published X-ray properties (such as $L_{x}$ and their spectral steepness) do not form a homogeneous set of data.

In order to construct a homogeneous data set for a meaningful statistical analysis, we normalized the data with the following procedures:

1. We adopted a simple absorbed power-law (PL) model for all the X-ray detected MSPs. We emphasize that the photon indices $\Gamma$ adopted in our analysis are not necessary reflecting the property of the non-thermal component as MSPs can also emit thermal X-rays from their heated polar caps (Cheng \& Zhang 1999). However, for modeling the thermal component, while some studies have adopted a simple black-body model, some other have adopted more sophisticated model such as atmospheric model. In order to avoid this inhomogeneity, we used a simple PL with its $\Gamma$ as a effective index to provide a convenient measure of the X-ray hardness with the interstellar absorption corrected.

2. Using absorption-corrected X-ray fluxes $f_{x}$ and $\Gamma$ in the band reported in literature and their corresponding statistical uncertainties, we computed $f_{x}$ and their errors for the X-ray detected MSPs in an energy range of $2-10 \mathrm{keV}$ with the aid of PIMMS. ${ }^{2}$

3. For the distances $d$ used in calculating $L_{x}=$ $4 \pi d^{2} f_{x}$, more than half of the X-ray detected MSPs (25) have their estimates of $d$ derived from the dispersion measures $\left(d_{D M}\right)$ only (Manchester et al. 2005). For the MSPs which have their $d$ estimated by dedicated investigations (e.g. parallax), these values are adopted instead of those derived from dispersion measures as they are more reliable. We noted that most of our sample do not have any uncertainty estimate of $d$. To provide an overall uncertainty estimate of $d$, we constructed the distribution for the relative difference between $d_{D M}$ and those determined by more accurate methods $\left(d-d_{D M}\right) / d_{D M}$ of the entire pulsar population. The standard deviation of this distribution is found to be 0.41 . This is consistent with the uncertainty of $\pm 40 \%$ as adopted by Possenti et al. (2002). In this study, we assume a percentage error of $\pm 41 \%$ for all our adopted distance which is subsequently propagated into the error budget of $L_{x}$.

4. We re-analysed the X-ray data of the MSPs and computed the $1 \sigma$ uncertainties of $\Gamma$ and $L_{X}$ for the following cases: (i) the X-ray properties of the MSPs are not modeled with a single PL in the existing literature (ii) there is no error estimation for either $\Gamma$ or $L_{x}$ in the existing literature. For each of these MSPs, their background-subtracted X-ray spectra were fitted with an absorbed PL model by using XSPEC. For the MSPs with the spectral models different from a simple absorbed PL in literatures, we have compared their $f_{x}$ obtained in our analysis and their values reported in the corresponding literatures. And we found the differences are all less than $2 \sigma$ of the flux uncertainties.

In re-examining the XMM-Newton data of PSR J16003053 and PSR J1832-0836 (firstly reported by Prinz \& Becker 2015), we found the signal-to-noise ratios of the potential X-ray counterparts of these two MSPs are $2 \sigma$. In view of this, these two pulsars will be excluded from our list of confirmed X-ray detected MSPs as both $\Gamma$ and $L_{x}$ cannot be properly constrained.

In Table 1, we summarize the physical properties of 47 X-ray detected MSPs together with their $L_{x}$ and $\Gamma$ normalized by the aforementioned procedures. Our MSP

\footnotetext{
${ }^{2}$ https://heasarc.gsfc.nasa.gov/docs/software/tools/pimms.html
} 
sample size is almost five times larger than that adopted in Possenti et al. (2002).

We have also found that 21 other MSPs without any reported X-ray investigation have been serendipitously covered by the archival data obtained by Swift XRT. We have performed a systematic search for their X-ray counterparts by means of a wavelet source detection algorithm. For all these $21 \mathrm{MSPs}$, we do not yield any detection larger than $3 \sigma$. Estimating the $n_{H}$ of these MSPs by the total HI column density through the Galaxy at their radio timing positions (Kalberla et al. 2005) and assuming a photon index of $\Gamma=2$, we computed $1 \sigma$ upper limits of their $L_{x}$ in $2-10 \mathrm{keV}$ using their distances and limiting count rates. Together with the limiting luminosities of 13 other MSPs reported by other literatures and those of PSR J1600-3053 and PSR J1832-0836 obtained from our re-analysis, the results are summarized in Table 2.

\section{STATISTICAL ANALYSIS}

With the sample prepared by the aforementioned procedures, we performed a detailed statistical analysis of the X-ray properties of MSPs.

\subsection{Correlation $\& 3$ regression analysis}

We started by investigating whether $L_{X}$ is correlated with a number of proper-motion corrected derived parameters, including spin-down power $\dot{E}$, characteristic ages $\tau$, magnetic-field strength at the stellar surface $B_{s}$ and magnetic-field strength at the light cylinder $B_{L C}$.

We noted there is a large fraction of non-detections in our sample and these MSPs have relatively low $\dot{E}$ (see Table 2 and Figure 1), Ignoring these upper-limits will result in strong selection bias (i.e. Malmquist bias). For handling the censored data (i.e. 46 detections + 35 upper-limits), we have used the CRAN NADA package to perform the survival analysis ${ }^{3}$. Non-parametric approach has been adopted for both correlation and regression analysis.

We used the generalized Kendall's $\tau$ for testing the correlation (cf. Helsel 2005). The corresponding $p$-values are summarized in Table 3 . Strong positive correlations are found for $L_{x}-\dot{E}\left(p-\right.$ value $\left.=2.4 \times 10^{-4}\right)$ and $L_{x}-B_{L C}\left(p-\right.$ value $\left.=4.2 \times 10^{-4}\right)$. An anti-correlation between $L_{x}$ and $\tau\left(p\right.$-value $\left.=4.6 \times 10^{-3}\right)$ is also found. On the other hand, there is no evidence for the correlation between $L_{x}$ and $B_{s}(p-$ value $=0.97)$.

We proceeded with a linear regression analysis on the censored data to obtain an empirical $\log L_{x}-\log \dot{E}$ relation. The slope and the intercept are estimated by

\footnotetext{
${ }^{3}$ https://CRAN.R-project.org/package $=$ NADA
}

the Akritas-Thiel-Sen (ATS) line (Akritas et al. 1995) and the Turnbull estimate (Turnbull 1976) respectively. The $1 \sigma$ errors of the model parameters are estimated by bootstrap resampling with 1500 simulated datasets.

The best-fit relation is found to be

$$
\log L_{x}=(1.31 \pm 0.22) \log \dot{E}-(14.80 \pm 7.68)
$$

Figure 1 clearly shows the positive correlation between $L_{x}$ of MSPs and their $\dot{E}$. The ATS fit is illustrated by a solid line. We have also carried out the standard linear regression with the X-ray detected MSPs only. This yields $\log L_{x}=1.26 \log \dot{E}-12.53$. Together with the relation estimated by Possenti et al. (2002) with 10 MSPs, we overplot these two lines in Figure 1 for comparison. One should note that the ATS estimate lies below the other two lines. This demonstrates that the results of regression analysis without taking the upper-limits into account tend to overestimate the $\mathrm{X}$-ray conversion efficiency.

Applying the same procedures on the censored data, we have also obtained the best-fit relation for $L_{x}-B_{L C}$ :

$$
\log L_{x}=(1.63 \pm 0.30) \log B_{L C}+(22.12 \pm 1.45)
$$

and for $L_{x}-\tau$ :

$$
\log L_{x}=-(1.53 \pm 0.42) \log \tau+(44.75 \pm 4.08) .
$$

\subsection{Searches for differences among various $M S P$ classes}

As MSPs can now be divided into different classes, it is interesting to compare their properties. In our study, we divided our sample of X-ray detected MSPs into four classes: (1) RBs, (2) BWs, (3) Isolated MSP and (4) Others. The classification of RBs and BWs is based on the online catalog provided by Alessandro Patruno ${ }^{4}$. For the MSPs which do not have any identified companions in the ATNF catalog (Manchester et al. 2005), we put them in the category of isolated MSPs. For those not belonging to classes (1), (2) or (3), we put them into the forth category of "Others". The classes of each X-ray detected MSP are specified in Table 1.

To compare the properties among different MSP classes, we focus on the following parameters: $L_{x}, \Gamma, \tau$, $B_{L C}, B_{s}, \dot{E}$ and $P_{b}$.

We firstly constructed the cumulative distribution functions of the aforementioned parameters, which are shown in Figures 2-8. For searching the possible differences among these classes, we apply a non-parametric

\footnotetext{
4 https://apatruno.wordpress.com/about/millisecond-pulsarcatalogue/
} 
two-sample Anderson-Darling (A-D) test (Anderson \& Darling 1952; Darling 1957; Pettitt 1976, Scholz \& Stephens 1987) to their unbinned distributions. We have also compared the results of A-D test with those obtained from the conventional Kolmogorov-Smirnov (KS) test. The results of both A-D test and K-S test are summarized in Table 4.

Among all the tested parameters, we found the distributions of $B_{s}$ of different classes are comparable. On the other hand, the distributions of $B_{L C}$ and $\dot{E}$ for RBs are significantly different from those of "Others" $\left(p\right.$-value $=0.01$ and 0.002 for $B_{L C}$ and $\dot{E}$ respectively $)$ and marginally different from that of isolated MSPs $\left(p\right.$-value $=0.061$ and 0.053 for $B_{L C}$ and $\dot{E}$ respectively). Both $B_{L C}$ and $\dot{E}$ of RBs are found to be generally higher than those of isolated MSPs and "Others".

In comparing the distributions of characteristic ages $\tau$, RBs are found to be younger than "Others" ( $p$-value 0.0035). On the other hand, there is a marginal indication that BWs are younger than "Others" ( $p$-value $0.04)$.

The significances of the aforementioned results are unaltered when the statistical uncertainties of the pulsar parameters given by the ATNF catalog are taken into account.

We have also compared the distributions of the orbital period $P_{b}$ for RBs, BWs and "Others". The $P_{b}$ of Both RBs and BWs are significantly shorter than "Others" $\left(p\right.$-value $\left.<5 \times 10^{-4}\right)$, as expected given the selection criteria. On the other hand, there is no strong evidence that the $P_{b}$ distributions of RBs and BWs are different $(p$-value $=0.1)$.

While the distributions of the parameters (i.e. $\tau, B_{s}$, $B_{L C}, \dot{E}$ and $P_{b}$ ) of RBs are comparable with those of BWs, their X-ray properties appears to be rather different. In comparing the $L_{x}$ and $\Gamma$ distributions of these two classes, A-D tests yield the $p$-values of 0.003 and 0.01 respectively. This suggests the $\mathrm{X}$-ray emission of RBs is brighter and harder than that of BWs.

We would like to point out that the significances of their differences in $L_{x}$ and $\Gamma$ can be reduced considerably ( $p$-value $=0.7$ and 0.2 respectively) by taking into account the statistical uncertainties of the X-ray parameters.

\section{SUMMARY \& DISCUSSION}

We have carried out a census for the X-ray population of MSPs and performed a detailed statistical analysis of their physical and X-ray properties. $L_{x}$ is found to be positively correlated with $\dot{E}$ and $B_{L C}$. There is also evidence of $L_{x}$ decreasing with $\tau$. The best-fit $L_{x}-\dot{E}$ relation of MSPs are found to be $L_{x} \simeq 10^{31.05} \dot{E}_{35}^{1.31} \mathrm{erg} / \mathrm{s}$ in $2-10 \mathrm{keV}$, where $\dot{E}$ is the spin-down power in units of $10^{35} \mathrm{erg} / \mathrm{s}$.

In comparing the properties among different classes of MSPs, we found that their distributions of $B_{s}$ are comparable. However, $B_{L C}$ and $\dot{E}$ of RBs are significantly higher than those of "Others". There is also some marginal evidence that RBs and BWs are younger than "Others".

While the pulsar and orbital parameters of RBs and BWs are comparable, $L_{x}$ of RBs are found to be higher than those of BWs. Also, we have found the indication that the X-ray emission of RBs is harder than that of BWs in 2-10 keV though a firm conclusion is limited by their statistical uncertainties.

In the following, we discuss the theoretical implication of these findings with a specific focus on RBs and BWs.

In MSP binary systems, there are three regions to produce the X-rays, which are intra-binary shock, magnetosphere and heated polar cap. In the hypothesis that the $\mathrm{GeV}$ gamma-rays are produced in the outer magnetosphere around the light cylinder (Aliu et al. 2008; Abdo et al. 2013). the photon-photon paircreation process makes a secondary pair. The synchrotron radiation of the secondary pairs can produce the synchrotron photon with a typical photon energy of $E_{\text {syn }} \sim 0.7\left(\gamma_{s} / 2 \cdot 10^{3}\right)^{2}\left(B / 10^{5} \mathrm{G}\right)(\sin \theta / 0.1) \mathrm{keV}$, where $\gamma_{s}$ is the Lorentz factor of the secondary, $B$ is the magnetic field strength at the light cylinder and $\theta$ is the pitch angle (Takata et al. 2012). Based on the outer gap accelerator model, Takata et al. (2012) discuss that the magnetospheric synchrotron luminosity is related to the spin down power as $L_{\text {syn }} \sim 6 \times 10^{30}\left(\dot{E} / 10^{35} \mathrm{erg} \mathrm{s}^{-1}\right)^{35 / 32}$, which is one or two orders of magnitude smaller than the observed X-ray emission from the BW/RB systems (green lines in Figures 9 and 11).

For the observed pulsed X-ray emission of the FermiLAT MSPs, the magnetospheric emission dominates in the spectral energy distribution of the MSPs with a higher spin down power, say $\dot{E}>10^{36} \mathrm{erg} \mathrm{s}^{-1}$ ( $\mathrm{Ng}$ et al. 2014). For the MSPs with a lower spin down power, on the other hand, the heated polar cap emission dominates the magnetospheric emission in the spectrum. As we can see in Figures 9 and 11, the binary systems with a smaller spin down power show a tendency to have very soft X-ray emission with an effective photon index $\Gamma>3$. This index is difficult to be explained by a standard synchrotron emission from the shock with a typical power law index of the accelerated pulsar wind particles, and it suggests a contribution of the emission from the heated polar cap region.

The heated polar cap emission from MSPs are observed with two components (Bogdanov \& Grindlay 
2009); (i) rim component with a temperature of $T_{r} \sim$ $5 \times 10^{5} \mathrm{~K}$ and an effective radius of $R_{r} \sim 3 \mathrm{~km}$, and (ii) core component with a temperature of $T_{c} \sim 2 \times 10^{6} \mathrm{~K}$ and an effective radius of $R_{c} \sim 0.1 \mathrm{~km}$. In Takata et al. (2012), the two temperatures are modeled as

$$
T_{r} \sim 5.2 \times 10^{5} \dot{E}_{35}^{7 / 96} B_{8}^{5 / 48} R_{r, 3}^{-1 / 2} \mathrm{~K}
$$

and

$$
T_{c} \sim 3.5 \times 10^{6} \dot{E}_{35}^{3 / 32} B_{8}^{1 / 16} R_{c, 0.1}^{-1 / 2} \mathrm{~K}
$$

respectively, where $\dot{E}_{35}=\dot{E} / 10^{35} \mathrm{erg} \mathrm{s}^{-1}, R_{r, 3}=$ $R_{r} / 3 \mathrm{~km}$ and $R_{c, 0.1}=R_{c} / 0.1 \mathrm{~km}$. This heated polar cap emission will dominate the shock emission in the observation of the BW with a lower spin down power (red lines in Figures 9).

To model the shock emission, we use a simple onezone model and calculate the synchrotron radiation of the accelerated electrons/positrons in the pulsar wind. We assume that the shock is located close to the companion star, at a distance from the MSP $r_{s} \sim 10^{11} \mathrm{~cm}$ is the typical separation between two stars. We evaluate the magnetic field of the pulsar wind at the shock from $B_{s}=3\left(\dot{E} \sigma_{P W} a / r_{s}^{2} c\right)^{1 / 2} \sim$ $17 \dot{E}_{35}^{1 / 2}\left(\sigma_{P W} / 0.1\right)^{1 / 2}\left(r_{s} / 10^{11} \mathrm{~cm}\right)^{-1} \mathrm{G}$ (Kennel \& Coroniti 1984), where $\sigma_{P W}$ is the ratio of the magnetic energy to the kinetic energy of the cold relativistic pulsar wind. To compare with the population of BWs and $\mathrm{RBw}$, we calculate the shock emission with $\sigma_{P W}=0.1$, at which the synchrotron emission from the shocked pulsar wind becomes maximum, and $r_{s}=10^{11} \mathrm{~cm}$. At the shock, the electrons/positrons are accelerated beyond the Lorentz factor $\left(\Gamma_{1}\right)$ of the cold relativistic pulsar wind, and forms a power law distribution in the energy. We assume that $\Gamma_{1}=10^{5}$ in the calculation. We determine the maximum Lorentz factor of the accelerated particles by balancing the accelerating time scale, $\tau_{a c c}=\Gamma m_{e} c /\left(e B_{s}\right)$, and the synchrotron cooling time scale $\tau_{s} \sim 9 m_{e} c^{3} c^{5} /\left(4 e^{4} B_{s}^{2} \Gamma\right)$, that is, $\Gamma_{\text {max }} \sim 2.7 \times 10^{7}\left(B_{s} / 20 \mathrm{G}\right)^{-1 / 2}$. For the initial distribution, we assume the hard power low index $p=1.5$ to explain the hard spectrum of the X-ray emissions from some binary systems. We solve the evolution of the distribution function under the synchrotron energy loss.By assuming the speed of the post shocked flow is constant, we calculate the magnetic field evolution from the magnetic flux conservation $B=B_{s}\left(r_{s} / r\right)$ (Kennel \& Coroniti 1984).

The shock luminosity is proportional to $L_{X} \propto \delta \dot{E}$, where $\delta$ is the fraction of the pulsar wind blocked by the outflow from the companion star and/or the companion star itself. While the pulsar and orbital parameters of BWs and RBs are comparable, the X-ray luminosity of the RBs is significantly higher than that of the
BWs. This can be explained by the difference in the fraction $\delta$. We may estimate the fraction of the sky intercepted by the companion star with $\delta \sim\left(R_{R} / 2 a\right)^{2}$, where $R_{R}$ is the Roche-lobe radius of the companion star. Since the Roche-lobe radius is estimated as $R_{R} / a=0.462[q /(1+q)]^{1 / 3}$ with $q$ being the ratio of the companion mass to the neutron star mass (Frank et al. 2002), the fraction becomes $\delta=0.053[q /(1+q)]^{2 / 3}$. With the typical values of the mass ratio, we estimate $\delta \sim 1 \%$ for $\operatorname{RB}(q=0.1)$ and $\delta \sim 0.2 \%$ for the $\mathrm{BW}$ $(q=0.01)$. For most RBs, moreover, the companion star has been identified as a low-mass (G/M type) main-sequence star (see ATNF catalog, Manchester et al. 2005). In such system, a larger fraction could be realized by the magnetized outflow from the companion star (Archibald et al. 2013). Since the companion star is tidally locked, the spin period is equal to the orbital period of several hours. The low mass, rapidly spinning main sequence star can have a surface magnetic field of several hundreds to a few thousand Gauss (Reiners et al. 2009). Since the shock distance will be located at several stellar radius of the companion star, the magnetized outflow from the companion star may overcome the pulsar wind. We may assume the radial dependence of the stellar magnetic field as $B(R)=B_{*}\left(R_{*} / R\right)^{m}$, where $B_{*}$ and $R_{*}$ are stellar magnetic field and radius, respectively, and $R$ is the distance from the center of the star. The magnetic field in the stellar wind could deviate from the dipole field $(m=3)$ and have $m<3$, owing to the spin of the star and/or more complicate surface magnetic field structure (Banaszkiewicz et al. 1998). The typical distance to the shock from the pulsar, $r_{s}$, may be estimated from the momentum balance of $B^{2}(R) c=L_{s d} / r_{s}$, where $r_{s}=a-R$. Then we would estimate required surface magnetic field strength to overcome the pulsar wind from the condition that $r_{s}<a / 2$. Under aforementioned magnetic field configuration, we have $B_{*}>(a / 2)^{m-1} R_{*}^{-m}\left(L_{s d} / c\right)^{1 / 2}$, which yields $B_{*} \gtrsim 500 \mathrm{G}$ for the dipole field $(m=3)$ and $B_{*} \gtrsim 200 \mathrm{G}$ for $m=2$ with $a=10^{11} \mathrm{~cm}, L_{s d}=10^{35} \mathrm{erg} / \mathrm{s}$ and $R_{*}=0.3 R_{\odot}$. Hence, the magnetized stellar wind will be able to overcome the pulsar wind if the stellar magnetic field can be enhanced to $B_{*}>10^{2-3} \mathrm{G}$.

It has been observed that the low frequency radio wave for the RB is observed with an eclipse lasting a large part of the orbital phase. This also suggests that a large fraction of the pulsar's sky is blocked by the outflow from the companion stars.

The predicted luminosity and photon index from BW and RB are summarized in the Figures $9 \sim 12$; In each figure, the blue lines show the model prediction for the relation between the X-ray luminosity and spin down 
power (left panels) or the photon index (right panels), and they consider the contribution from the shock emission and the heated polar cap emission. In Figure 9 and 11 , we consider the different fraction, $\delta$, for the shock emission; the solid, dashed and dotted lines are results for $\delta=0.15 \%, 0.3 \%$ and $0.6 \%$, respectively. We can see in the figure that for a fixed X-ray luminosity, the calculated photon index is softer for a smaller efficiency, $\delta$. This is because the contribution of the shock emission relatively to the heated polar cap emission becomes smaller. For the BW, our model predicts that the X-ray emission for the spin down power $\dot{E}<10^{34} \mathrm{erg} \mathrm{s}^{-1}$ is dominated by the heated polar cap emission. This can explain the observed soft spectrum of some BW pulsars. As Figure 11 shows, the current model predicts that the observed X-ray emissions from the RB is dominated by the shock emission, and the contribution of the heated polar cap emission is negligible, except for PSR J1816+4516, for which the heated polar cap emission may be observed. In Figures 10 and 12, we summarize the dependency on the surface magnetic field, which affects the heated polar cap temperature through the equations (4) and (5); the solid, dashed and dotted lines are results for $B_{s}=1.5 \times 10^{8} \mathrm{G}, 3 \times 10^{8} \mathrm{G}$ and $6 \times 10^{8} \mathrm{G}$, respectively. We can see in the figures that for a fixed spin down power, the predicted X-ray emission shows a less dependency on the surface magnetic field. With a reasonable range of the parameters of the MSPs, the current model is qualitatively consistent with the observations. Based on our result, we suggest the pulsation search for the BWs with a lower spin down power, for which the emission will be dominated by the heated polar cap emission, since an increase in the sample of the pulse profiles of the heated polar cap will be useful to study the equation of the state for the neutron star.

JL is supported by BK21 plus Chungnam National University and the National Research Foundation of Korea grant 2016R1A5A1013277; CYH is supported by the National Research Foundation of Korea grant 2016R1A5A1013277; JT is supported by the NSFC grants of China under 11573010, U1631103 and 11661161010; AKHK by the Ministry of Science and Technology of Taiwan grants 106-2918-I-007-005 and 105-2112-M-007-033-MY2; PHT is supported by the National Natural Science Foundation of China (NSFC) grants 11633007 and 11661161010; KSC are supported by GRF grant under 17302315 . 
Abdo, A. A., et al. 2013, ApJS, 208, 17

Akritas, M.G., Murphy, S. A., \& LaValley, M. P. 1995, J.

Amer. Stat. Assn., 90, 170

Aliu,E., Anderhub,H., Antonelli,L.A., Antoranz,P., Backes,M. et al. 2008, Sci, 322, 1221

Alpar, M. A., Cheng, A. F., Ruderman, M. A., \& Shaham, J. 1982, Nature, 300, 728

Anderson, T.W., \& Darling, D.A. 1952, Annals of Mathematical Statistics, 23, 193

Aoki, Y., et al. 2012, IAUS, 279, 317

Archibald, Anne M., et al. 2009, Science, 324, 1411

Archibald, Anne M., et al. 2010, ApJ, 722, 88

Archibald, Anne M., et al. 2013, arXiv1311.5161

Arumugasamy, P., Pavlov, G. G., Garmire, G. P. 2015, ApJ, 814, 90

Backer, D. C., Kulkarni, S. R., Heiles, C., Davis, M. M., \& Goss, W. M. 1982, Nature, 300, 615

Banaszkiewicz, M., Axford, W. I., \& McKenzie, J. F. 1998, A\&A, 337, 940

Becker, W., \& Trumper, J. 1997, A\&A, 326, 682

Bogdanov, S., Grindlay, J. E. 2009, ApJ, 703, 1557

Bogdanov, S., et al. 2011, ApJ, 742, 97

Bogdanov, S., et al. 2014, ApJ, 789, 40

Cheng, K. S., \& Zhang, L. 1999, ApJ, 515, 337

Darling, D.A. 1957, Annals of Mathematical Statistics, 28, 823

Espinoza, C. M., et al. 2013, MNRAS, 430, 571

Fabian, A. C., Pringle, J. E., Verbunt, F., \& Wade, R. A. 1983, Nature, 301, 222

Frank, J., King, A., Raine, D. 2002, Accretion Power in Astrophysics (Cambridge: Cambridge Univ. Press)

Fruchter, A. S., Stinebring, D. R., \& Taylor, J. H. 1988, Nature, 333, 237

Gentile, P. A., et al. 2014, ApJ, 793, 69

Helsel, D.R. 2005, Nondetects and Data Analysis: Statistics for censored environmental data, Wiley-Interscience, New York

Huang, R. H. H., et al. 2012, ApJ, 760, 92

Hui, C. Y., Cheng, K. S., \& Tamm, Ronald E. 2010, ApJ, 714,1149

Hui, C. Y. 2014, JASS, 31, 101

Hui, C. Y., et al. 2014, ApJ, 781, 21

Hui, C. Y., et al. 2015, ApJ, 801, 27

Kalberla, P. M. W., et al. 2005, A\&A, 550, 775

Kargaltsev, O., \& Pavlov, G. G. 2008, in AIP Conf. Proc. 983, 40 Years of Pulsars: Millisecond Pulsars, Magnetars and More, ed. C. Bassa et al. (Melville, NY: AIP), 171
Kennel, C. F., \& Coroniti, F. V. 1984, ApJ, 283, 694

Li, K.L., Kong, A.K.H, Takata, J., et al. 2014, ApJ, 797, 111

Manchester, R. N., Hobbs, G. B., Teoh. A., \& Hobbs, M. 2005, ApJ, 129, 1993

Marelli, M., De Luca, A., \& Caraveo, P. A. 2011, ApJ, 733, 82

Homer, Lee, et al. 2007, AJ, 131, 562

Ng, C.-Y., Takata, J., Leung, G. C. K., Cheng, K. S., Philippopoulos, P., 2014, ApJ, 787, 167

Nicastro, L., et al. 2004, A\&A, 413, 1065

Papitto, A., Ferrigno, C., Bozzo, E., et al. 2013, Nature, 501, 517

Pavlov, G. G., Kargaltsev, O., Garmire, G. P., \& Wolszczan, A. 2007, ApJ, 664, 1072

Pettitt, A.N. 1976, Biometrika, 63, 161

Possenti, A., Cerutti, R., Colpi, M., \& Mereghetti, S. 2002, A\&A, 387, 993

Pancrazi, B., et al. 2012, A\&A, 544, 108

Prinz, T., \& Becker, W. 2015arXiv:151107713

Radhakrishnan, V., \& Srinivasan, G. 1982, CScience, 51, 1096

Ray, P. S., et al. 2012arXiv1205.3089

Reiners, A.; Basri, G.; Browning, M., 2009, ApJ, 692, 538

Scholz, F.W., \& Stephen, M.A. 1987, Journal of the

American Statistical Association, 82(339), 918

Seward, F. D., \& Wang, Z. 1988, ApJ, 332, 199

Shibata, S., Watanabe, E., Yatsu, Y., Enoto, T., \& Bamba, A. 2016, ApJ, 833, 59

Spiewak, R., et al. 2016, ApJ, 822, 37

Stappers, B. W., Archibald, A. M., Hessels, J. W. T., et al. 2014, ApJ, 790, 390

Strader, J., et al. 2015, ApJ, 804, 12

Takata, J., Li, K. L., Leung, G. C. K., et al. 2014, ApJ, 785,131

Takata, J., Cheng, K.S. Taam, R.E., 2012, ApJ, 745, 100

Turnbull, B.W. 1976, Journal of the Royal Statistical

Society Series B-Methodological, 38(3), 290

van den Heuvel, E. P. J., \& van Paradijs, J. 1988, Nature, 334,227

Vink, J., Bamba, A., \& Yamazaki, R. 2011, ApJ, 727, 131

Yatsu, Y., 2015, ApJ, 802, 84

Zavlin, V. E., et al. 2006, ApJ, 638, 951 
Table 1. Pulsar parameters and X-ray properties of $47 \mathrm{X}$-ray detected MSPs. $\dot{E}, \tau, B_{\mathrm{s}}$ and $B_{\mathrm{LC}}$ are derived from the proper-motion corrected period derivatives.

\begin{tabular}{|c|c|c|c|c|c|c|c|c|c|c|c|c|}
\hline MSP name & Inst. & Class & $\begin{array}{c}P \\
\mathrm{~ms}\end{array}$ & $\begin{array}{c}P_{b} \\
\text { day } \\
\end{array}$ & $\begin{array}{l}\log \dot{E} \\
\mathrm{erg} / \mathrm{s}\end{array}$ & $\begin{array}{r}d \\
\mathrm{kpc}\end{array}$ & $\begin{array}{c}\tau \\
\text { Gyr } \\
\end{array}$ & $\begin{array}{c}B_{\mathrm{S}} \\
10^{8} \mathrm{G} \\
\end{array}$ & $\begin{array}{c}B_{\mathrm{LC}} \\
10^{4} \mathrm{G} \\
\end{array}$ & $\begin{array}{c}\log L_{x}(2-10 \mathrm{keV}) \\
\mathrm{erg} / \mathrm{s}\end{array}$ & $\Gamma$ & References \\
\hline J0023+0923 & $\mathrm{C}$ & BW & 3.05 & 0.14 & 34.13 & $1.25 \pm 0.52$ & 5.03 & 1.73 & 5.55 & $29.35_{-0.53}^{+0.47}$ & $3.3 \pm 0.5$ & 1 \\
\hline $\mathrm{J} 0030+0451$ & $\mathrm{R}$ & I & 4.87 & .. & 33.53 & $0.36 \pm 0.15$ & 7.69 & 2.24 & 1.76 & $29.88_{-1.19}^{+0.84}$ & $2.0 \pm 0.2$ & 2 \\
\hline J0034-0534 & $\mathrm{X}$ & $\mathrm{O}$ & 1.88 & 1.589 & 34.38 & $1.35 \pm 0.56$ & 7.32 & 0.88 & 12.10 & $29.48_{-1.11}^{+0.78}$ & $2.75_{-0.70}^{+1.29}$ & 3 \\
\hline J0101-6422 & $\mathrm{C}$ & $\mathrm{O}$ & 2.57 & 1.788 & 33.93 & $1.00 \pm 0.41$ & 11.2 & 0.98 & 5.23 & $29.90_{-0.54}^{+0.38}$ & $3.25_{-0.55}^{+0.56}$ & 3,4 \\
\hline $\mathrm{J} 0218+4232$ & B & $\mathrm{O}$ & 2.32 & 2.029 & 35.38 & $3.15 \pm 1.30$ & 0.48 & 4.27 & 31.06 & $33.20_{-0.93}^{+0.62}$ & $0.94 \pm 0.22$ & 2 \\
\hline $\mathrm{J} 0337+1715$ & $\mathrm{x}$ & $\mathrm{O}$ & 2.73 & 1.629 & 34.53 & $1.30 \pm 0.54$ & 2.45 & 2.22 & 10.2 & $29.76_{-0.64}^{+0.47}$ & $3.6_{-0.8}^{+1.1}$ & 5 \\
\hline J0437-4715 & $\mathrm{R}$ & $\mathrm{O}$ & 5.76 & 5.741 & 33.45 & $0.16 \pm 0.07$ & 6.64 & 2.85 & 1.35 & $30.19_{-0.71}^{+0.51}$ & $2.35 \pm 0.35$ & 2 \\
\hline J0613-0200 & $\mathrm{X}$ & $\mathrm{O}$ & 3.06 & 1.199 & 34.09 & $0.78 \pm 0.32$ & 5.42 & 1.68 & 5.31 & $29.50_{-1.01}^{+0.67}$ & $2.7 \pm 0.4$ & 6 \\
\hline J0614-3329 & S & $\mathrm{O}$ & 3.15 & 53.59 & 34.34 & $0.62 \pm 0.26$ & 2.85 & 2.38 & 6.91 & $29.92_{-0.52}^{+0.35}$ & $2.63_{-0.27}^{+0.30}$ & 7 \\
\hline J0636+5129 & $\mathrm{x}$ & $\mathrm{O}$ & 2.87 & 0.07 & 33.75 & $0.20 \pm 0.08$ & 13.6 & 0.99 & 3.82 & $27.93_{-0.82}^{+1.18}$ & $5.0_{-1.0}^{+5.0}$ & 5 \\
\hline $\mathrm{J} 0751+1807$ & $\mathrm{R}$ & $\mathrm{O}$ & 3.48 & 0.26 & 33.75 & $1.11 \pm 0.46$ & 9.14 & 1.47 & 3.16 & $31.29_{-1.08}^{+0.85}$ & $2.0 \pm 0.2$ & 2 \\
\hline $\mathrm{J} 1012+5307$ & $\mathrm{R}$ & $\mathrm{O}$ & 5.26 & 0.61 & 33.49 & $0.70 \pm 0.29$ & 7.39 & 2.6 & 1.54 & $29.58_{-1.19}^{+0.83}$ & $2.3 \pm 0.2$ & 2 \\
\hline $\mathrm{J} 1023+0038$ & $\mathrm{C}$ & $\mathrm{RB}$ & 1.96 & 0.20 & 34.4 . & $1.37 \pm 0.57$ & 2.63 & 0.94 & 12.24 & $31.90_{-0.75}^{+0.31}$ & $1.19 \pm 0.03$ & 8 \\
\hline J1024-0719 & $\mathrm{R}$ & $\mathrm{O}$ & 5.16 & .. & 33.72 & $1.22 \pm 0.50$ & 4.41 & 3.13 & 2.13 & $29.09_{-1.25}^{+0.80}$ & $2.0 \pm 0.2$ & 2 \\
\hline J1124-3653* & $\mathrm{C}$ & BW & 2.41 & $\cdot$. & 33.6 & $1.05 \pm 0.43$ & 27.05 & 0.59 & 3.88 & $30.56_{-0.68}^{+0.45}$ & $2.1 \pm 0.3$ & 9 \\
\hline $\mathrm{J} 1227-4853$ & $\mathrm{C}, \mathrm{X}$ & $\mathrm{RB}$ & 1.69 & 0.29 & 34.96 & $1.80 \pm 0.74$ & 2.41 & 1.38 & 27.1 & $32.12_{-0.47}^{+0.31}$ & $1.2 \pm 0.04$ & 10 \\
\hline $\mathrm{J} 1300+1240$ & $\mathrm{C}$ & $\mathrm{O}$ & 6.22 & 25.26 & 33.70 & $0.60 \pm 0.25$ & 3.23 & 4.41 & 1.67 & $28.82_{-0.56}^{+0.38}$ & $2.75 \pm 0.35$ & 11 \\
\hline $\mathrm{J} 1311-3430$ & $\mathrm{C}$ & BW & 2.56 & 0.07 & 34.69 & $2.43 \pm 1.00$ & 1.94 & 2.34 & 13.1 & $31.63_{-0.54}^{+0.37}$ & $1.3 \pm 0.3$ & 1 \\
\hline $\mathrm{J} 1417-4402$ & $\mathrm{C}$ & $\mathrm{O}$ & 2.66 & 5.37 &.. & $4.40 \pm 1.82$ &.. &.. &.. & $33.14_{-0.61}^{+0.38}$ & $1.32 \pm 0.4$ & 12 \\
\hline J1446-4701 & $\mathrm{X}$ & BW & 2.19 & 0.28 & 34.56 & $1.57 \pm 0.65$ & 3.61 & 1.47 & 12.73 & $30.30_{-0.75}^{+0.72}$ & $2.9_{-0.4}^{+0.5}$ & 1 \\
\hline J1514-4946 & $\mathrm{C}$ & $\mathrm{O}$ & 3.59 & 1.92 & 33.99 & $0.91 \pm 0.38$ & 4.94 & 2.06 & 4.04 & $29.37_{-0.61}^{+0.46}$ & $2.98_{-0.99}^{+1.2}$ & 3 \\
\hline $\mathrm{J} 1614-2230$ & $\mathrm{X}$ & $\mathrm{O}$ & 3.15 & 8.69 & 33.70 & $0.70 \pm 0.29$ & 12.5 & 1.13 & 3.30 & $27.55_{-0.56}^{+0.35}$ & $4.64_{-0.88}^{+1.50}$ & 13 \\
\hline J1628-3205* & $\mathrm{C}$ & $\mathrm{RB}$ & 3.21 & 0.21 & $34.26^{+}$ & $1.22 \pm 0.50$ & 3.38 & 2.23 & 6.20 & $30.96_{-0.50}^{+0.33}$ & $1.88_{-0.19}^{+0.20}$ & 3 \\
\hline $\mathrm{J} 1640+2224$ & $\mathrm{C}$ & $\mathrm{O}$ & 3.16 & 175.46 & 33.20 & $1.50 \pm 0.62$ & 39.0 & 0.64 & 1.85 & $29.64_{-0.55}^{+0.38}$ & $3.02_{-0.46}^{+0.50}$ & 3 \\
\hline J1658-5324 & $\mathrm{C}$ & I & 2.44 &.. & 34.48 & $0.88 \pm 0.36$ & 3.50 & 1.66 & 10.38 & $29.64_{-0.56}^{+0.39}$ & $3.22_{-0.64}^{+0.65}$ & 3,4 \\
\hline $\mathrm{J} 1709+2313$ & $\mathrm{C}$ & $\mathrm{O}$ & 4.63 & 22.71 & 32.63 & $2.18 \pm 0.90$ & 68.5 & 0.713 & 0.65 & $30.05_{-0.62}^{+0.44}$ & $1.49_{-0.80}^{+0.86}$ & 3 \\
\hline J1723-2837 & $\mathrm{C}, \mathrm{X}$ & $\mathrm{RB}$ & 1.86 & 0.62 & 34.67 & $0.72 \pm 0.30$ & 3.9 & 1.2 & 17.6 & $31.92_{-0.48}^{+0.31}$ & $1.0 \pm 0.07$ & 14 \\
\hline J1730-2304 & $\mathrm{X}$ & I & 8.12 &.. & 33.02 & $0.62 \pm 0.26$ & 8.99 & 3.45 & 0.59 & $29.18_{-0.63}^{+0.48}$ & $2.7_{-0.5}^{+0.9}$ & 4 \\
\hline J1731-1847 & $\mathrm{C}$ & BW & 2.34 & 0.31 & 34.87 & $4.78 \pm 1.98$ & 1.53 & 2.42 & 17.12 & $30.99_{-0.80}^{+0.60}$ & $1.9_{-1.3}^{+1.5}$ & 1 \\
\hline J1744-1134 & $\mathrm{C}$ & I & 4.07 &.. & 33.63 & $0.40 \pm 0.17$ & 8.96 & 2.34 & 2.68 & $29.09_{-0.718}^{+0.51}$ & $2.0 \pm 0.2$ & 2 \\
\hline $\mathrm{J} 1810+1744^{*}$ & $\mathrm{C}$ & $\mathrm{BW}$ & 1.66 & 0.15 & 34.60 & $2.36 \pm 0.98$ & 5.72 & 0.88 & 17.80 & $30.68_{-0.52}^{+0.44}$ & $2.2 \pm 0.4$ & 1 \\
\hline $\mathrm{J} 1816+4510$ & $\mathrm{C}$ & $\mathrm{RB}$ & 3.19 & 0.36 & 34.71 & $4.36 \pm 1.80$ & 1.21 & 3.70 & 10.35 & $30.32_{-0.59}^{+0.51}$ & $2.76_{-0.69}^{+0.74}$ & 3,4 \\
\hline J1909-3744 & $\mathrm{C}$ & $\mathrm{O}$ & 2.95 & 1.53 & 34.64 & $1.14 \pm 0.47$ & 16.5 & 0.92 & 3.27 & $29.72_{-0.51}^{+0.36}$ & $3.02_{-0.39}^{+0.40}$ & 3 \\
\hline J1911-1114 & $\mathrm{X}$ & $\mathrm{O}$ & 3.63 & 2.72 & 33.97 & $1.07 \pm 0.44$ & 5.04 & 2.06 & 3.91 & $30.40_{-0.51}^{+0.34}$ & $1.89_{-0.19}^{+0.20}$ & 3 \\
\hline $\mathrm{J} 1939+2134$ & B & I & 1.56 & .. & 36.04 & $3.50 \pm 1.45$ & 0.24 & 4.09 & 98.08 & $32.78_{-0.53}^{+0.36}$ & $1.94_{-0.11}^{+0.13}$ & 15 \\
\hline $\mathrm{J} 1959+2048$ & $\mathrm{C}$ & $\mathrm{BW}$ & 1.61 & 0.38 & 35.00 & $1.73 \pm 0.72$ & 2.40 & 1.32 & 28.80 & $31.04_{-0.48}^{+0.32}$ & $1.96 \pm 0.12$ & 16 \\
\hline $\mathrm{J} 2017+0603$ & $\mathrm{C}$ & $\mathrm{O}$ & 2.9 & 2.20 & 34.11 & $1.40 \pm 0.58$ & 5.77 & 1.54 & 5.73 & $29.98_{-0.57}^{+0.40}$ & $2.75 \pm 0.71$ & 3,4 \\
\hline $\mathrm{J} 2043+1711$ & $\mathrm{C}$ & $\mathrm{O}$ & 2.38 & 1.48 & 34.08 & $1.25 \pm 0.52$ & 9.11 & 1.00 & 6.77 & $29.79_{-0.64}^{+0.46}$ & $3.03_{-2.28}^{+2.14}$ & 3 \\
\hline $\mathrm{J} 2047+1053^{*}$ & $\mathrm{C}$ & BW & 4.29 & 0.12 & 34.02 & $2.79 \pm 1.15$ & 3.24 & 3.04 & 3.54 & $30.99_{-0.70}^{+0.52}$ & $0.87 \pm 0.68$ & 1 \\
\hline J2051-0827 & $\mathrm{C}, \mathrm{X}$ & $\mathrm{BW}$ & 4.51 & 0.099 & 33.72 & $1.47 \pm 0.61$ & 5.89 & 2.37 & 2.34 & $28.62_{-0.70}^{+0.55}$ & $4.1 \pm 0.7$ & 1 \\
\hline $\mathrm{J} 2124-3358$ & $\mathrm{R}$ & I & 4.93 &.. & 33.38 & $0.41 \pm 0.17$ & 10.7 & 1.92 & 1.45 & $29.77_{-1.14}^{+0.62}$ & $2.0 \pm 0.2$ & 2 \\
\hline $\mathrm{J} 2129-0429^{*}$ & $\mathrm{X}$ & $\mathrm{RB}$ & 7.62 & 0.64 & 34.48 & $1.83 \pm 0.76$ & 0.36 & 16.20 & 3.37 & $31.79_{-0.51}^{+0.34}$ & $1.25 \pm 0.04$ & 17 \\
\hline $\mathrm{J} 2214+3000$ & $\mathrm{C}$ & $\mathrm{BW}$ & 3.12 & 0.42 & 34.22 & $0.60 \pm 0.25$ & 3.88 & 2.02 & 6.03 & $28.78_{-0.62}^{+0.48}$ & $3.8 \pm 0.4$ & 1 \\
\hline $\mathrm{J} 2215+5135$ & $\mathrm{C}$ & $\mathrm{RB}$ & 2.61 & 0.17 & 34.87 & $2.77 \pm 1.15$ & 1.24 & 2.99 & 15.80 & $31.92_{-0.61}^{+0.41}$ & $1.4 \pm 0.2$ & 9 \\
\hline $\mathrm{J} 2241-5236$ & $\mathrm{C}$ & BW & 2.19 & 0.15 & 34.40 & $0.96 \pm 0.40$ & 5.22 & 1.22 & 10.90 & $29.88_{-0.57}^{+0.42}$ & $2.8 \pm 0.4$ & 1 \\
\hline $\mathrm{J} 2256-1024^{*}$ & $\mathrm{C}$ & BW & 2.29 & 0.21 & 34.60 & $1.33 \pm 0.55$ & 3.00 & 1.69 & 12.92 & $30.08_{-0.53}^{+0.37}$ & $2.9 \pm 0.3$ & 1 \\
\hline J2339-0533 & $\mathrm{S}$ & $\mathrm{RB}$ & 2.88 & 0.19 & 34.04 & $1.10 \pm 0.45$ & 6.83 & 1.41 & 5.35 & $31.44_{-0.49}^{+0.33}$ & $1.32 \pm 0.08$ & 18 \\
\hline
\end{tabular}

Note-Inst: C, X, S, Sw, R, and B stand for Chandra, XMM-Newton, Suzaku, Swift, ROSAT, and BeppoSAX, respectively. — Class: I, BW, RB and O stand for isolated MSPs, black-widows, redbacks and others respectively. - References : (1)Arumugasamy et al. (2015), (2)Possenti et al. (2002), (3)this work, (4)Prinz et al. (2015), (5)Spiewak et al. (2016), (6)Marelli et al. (2011), (7)Aoki et al. (2012), (8)Bogdanov et al. (2011), (9)Gentile et al. (2014), (10)Bogdanov et al. (2014), (11)Pavlov et al. (2007), (12)Strader et al. (2015), (13)Pancrazi et al. (2012), (14)Hui et al. (2014), (15)Nicastro et al. (2004), (16)Huang et al. (2012), (17)Hui et al. (2015), (18)Yatsu et al. (2015). - $\dagger \dot{E}$ is obtained from Roberts et al. (2015). ${ }^{*} \dot{P}, B_{S}, B_{L C}$, and $\tau$ are deduced from $\dot{E}$ obtained in the corresponding references.

Pulsar Parameters of all the others are obtained from ATNF catalog (Manchester et al. 2005). 
Table 2. Upper limits of $L_{x}$ of 36 MSPs. $\dot{E}, \tau, B_{\mathrm{s}}$ and $B_{\mathrm{LC}}$ are derived from the proper-motion corrected period derivatives.

\begin{tabular}{|c|c|c|c|c|c|c|c|c|c|c|c|}
\hline MSP name & Inst. & Class & $\begin{array}{c}P \\
\mathrm{~ms}\end{array}$ & $\begin{array}{c}P_{b} \\
\text { day }\end{array}$ & $\begin{array}{l}\log \dot{E} \\
\mathrm{erg} / \mathrm{s}\end{array}$ & $\begin{array}{c}d \\
\mathrm{kpc}\end{array}$ & $\begin{array}{c}\tau \\
\text { Gyr }\end{array}$ & $\begin{array}{c}B_{\mathrm{s}} \\
10^{8} \mathrm{G}\end{array}$ & $\begin{array}{c}B_{\mathrm{LC}} \\
10^{4} \mathrm{G}\end{array}$ & $\begin{array}{c}\log L_{x}(2-10 \mathrm{keV}) \\
\mathrm{erg} / \mathrm{s}\end{array}$ & References \\
\hline $\mathrm{J} 0340+4130$ & $\mathrm{Sw}$ & I & 3.30 & .. & 33.87 & $1.60 \pm 0.66$ & 7.76 & 1.51 & 3.82 & $<30.99$ & 1 \\
\hline J0610-2100 & $\mathrm{Sw}$ & $\mathrm{BW}$ & 3.86 & 0.286 & 33.92 & $3.26 \pm 1.34$ & 51.20 & 0.69 & 1.09 & $<31.70$ & 2 \\
\hline J0645+5158 & $\mathrm{X}$ & I & 8.85 & .. & 32.35 & $0.80 \pm 0.33$ & 35.80 & 1.89 & 0.25 & $<29.74$ & 3 \\
\hline $\mathrm{J} 1022+1001$ & $\mathrm{Sw}$ & $\mathrm{O}$ & 16.45 & 7.81 & 32.45 & $1.13 \pm 0.46$ & 8.16 & 7.34 & 0.15 & $<30.270$ & 1 \\
\hline $\mathrm{J} 1048+2339$ & $\mathrm{Sw}$ & $\mathrm{RB}$ & 4.67 & 0.25 & 33.89 & $2.00 \pm 0.82$ & 3.68 & 3.10 & 2.77 & $<31.17$ & 1 \\
\hline J1103-5403 & $\mathrm{C}$ & I & 3.39 &.. & 33.57 & $1.68 \pm 0.69$ & 14.60 & 1.13 & 2.72 & $<30.41$ & 3 \\
\hline J1435-6100 & $\mathrm{Sw}$ & $\mathrm{O}$ & 9.35 & 1.36 & 33.08 & $2.81 \pm 1.15$ & 6.05 & 4.84 & 0.56 & $<31.88$ & 1 \\
\hline J1455-3330 & $\mathrm{Sw}$ & $\mathrm{O}$ & 7.99 & 76.18 & 33.25 & $1.01 \pm 0.41$ & 5.50 & 4.34 & 0.77 & $<30.65$ & 1 \\
\hline J1525-5545 & Sw & $\mathrm{O}$ & 11.36 & 0.99 & 33.54 & $3.14 \pm 1.29$ & 1.37 & 12.40 & 0.79 & $<31.11$ & 1 \\
\hline $\mathrm{J} 1544+4937$ & $\mathrm{Sw}$ & $\mathrm{BW}$ & 2.16 & 0.12 & 34.08 & $2.99 \pm 1.23$ & 11.70 & 0.81 & 7.50 & $<31.28$ & 1 \\
\hline J1600-3053 & $\mathrm{x}$ & $\mathrm{O}$ & 3.6 & 14.35 & 33.87 & $1.80 \pm 0.74$ & 6.53 & 1.79 & 3.50 & $<30.70$ & 1 \\
\hline J1643-1224 & $\mathrm{X}$ & $\mathrm{O}$ & 4.62 & 147.02 & 33.86 & $0.74 \pm 0.30$ & 4.06 & 2.92 & 2.69 & $<30.75$ & 3 \\
\hline $\mathrm{J} 1713+0747$ & $\mathrm{Sw}$ & $\mathrm{O}$ & 4.57 & 67.83 & 33.52 & $1.18 \pm 0.48$ & 9.04 & 1.94 & 1.84 & $<31.05$ & 2 \\
\hline J1719-1438 & $\mathrm{X}$ & $\mathrm{O}$ & 5.79 & 0.09 & 33.18 & $0.34 \pm 0.14$ & 12.30 & 2.10 & 0.98 & $<29.37$ & 3 \\
\hline $\mathrm{J} 1738+0333$ & $\mathrm{Sw}$ & $\mathrm{O}$ & 5.85 & 0.36 & 33.65 & $1.47 \pm 0.60$ & 4.11 & 3.68 & 1.67 & $<30.54$ & 1 \\
\hline $\mathrm{J} 1741+1351$ & $\mathrm{Sw}$ & $\mathrm{O}$ & 3.75 & 16.34 & 34.34 & $1.08 \pm 0.44$ & 2.05 & 3.33 & 5.75 & $<30.38$ & 1 \\
\hline J1745-0952 & $\mathrm{Sw}$ & $\mathrm{O}$ & 19.38 & 4.94 & 32.67 & $0.23 \pm 0.09$ & 3.56 & 13.10 & 0.16 & $<29.16$ & 1 \\
\hline $\mathrm{J} 1745+1017$ & $\mathrm{Sw}$ & BW & 2.65 & 0.73 & 33.68 & $1.21 \pm 0.50$ & 18.60 & 0.78 & 3.81 & $<30.57$ & 1 \\
\hline J1748-3009 & $\mathrm{C}$ & $\mathrm{O}$ & 9.68 & 2.93 & .. & $5.07 \pm 2.08$ &.. & .. & .. & $<32.83$ & 3 \\
\hline $\mathrm{J} 1751-2857$ & $\mathrm{C}, \mathrm{X}$ & $\mathrm{O}$ & 3.91 & 110.75 & 33.84 & $1.09 \pm 0.45$ & 5.94 & 2.05 & 3.11 & $<30.81$ & 3 \\
\hline J1804-2717 & $\mathrm{Sw}$ & $\mathrm{O}$ & 9.34 & 11.13 & 33.24 & $0.80 \pm 0.33$ & 4.17 & 5.83 & 0.65 & $<30.09$ & 1 \\
\hline J1811-2405 & $\mathrm{Sw}$ & $\mathrm{O}$ & 2.66 & 6.27 & 34.42 & $1.83 \pm 0.75$ & 3.15 & 1.84 & 8.88 & $<31.40$ & 1 \\
\hline J1832-0836 & $\mathrm{X}$ & I & 2.72 &.. & 34.23 & $0.81 \pm 0.33$ & 5.0 & 1.55 & 7.22 & $<30.40$ & 1 \\
\hline J1843-1113 & $\mathrm{X}$ & I & 1.85 & .. & 34.77 & $1.26 \pm 0.52$ & 3.09 & 1.34 & 19.36 & $<30.62$ & 3 \\
\hline $\mathrm{J} 1850+0124$ & Sw & $\mathrm{O}$ & 3.56 & 84.95 & 33.98 & $3.39 \pm 1.39$ & 5.18 & 1.99 & 4.14 & $<32.35$ & 1 \\
\hline $\mathrm{J} 1853+1303$ & $\mathrm{X}$ & $\mathrm{O}$ & 4.09 & 115.65 & 33.69 & $1.32 \pm 0.54$ & 7.57 & 1.89 & 2.51 & $<30.64$ & 3 \\
\hline $\mathrm{J} 1857+0943$ & $\mathrm{x}$ & $\mathrm{O}$ & 5.36 & 12.33 & 33.65 & $1.20 \pm 0.49$ & 4.92 & 3.08 & 1.82 & $<30.61$ & 3 \\
\hline $\mathrm{J} 1900+0308$ & $\mathrm{Sw}$ & $\mathrm{O}$ & 4.91 & 12.48 & 33.30 & $4.80 \pm 1.97$ & 13.20 & 1.72 & 1.37 & $<32.49$ & 1 \\
\hline $\mathrm{J} 1901+0300$ & $\mathrm{Sw}$ & $\mathrm{O}$ & 7.80 & 2.40 & 33.58 & $5.29 \pm 2.17$ & 2.70 & 6.04 & 1.19 & $<31.94$ & 1 \\
\hline J1903-7051 & $\mathrm{Sw}$ & $\mathrm{O}$ & 3.60 & 11.05 & 33.82 & $0.93 \pm 0.38$ & 5.46 & 1.69 & 3.29 & $<30.45$ & 1 \\
\hline J1933-6211 & $\mathrm{C}$ & $\mathrm{O}$ & 3.54 & 12.82 & 33.43 & $0.65 \pm 0.27$ & 15.20 & 1.05 & 2.15 & $<30.28$ & 3 \\
\hline $\mathrm{J} 1943+2210$ & $\mathrm{Sw}$ & $\mathrm{O}$ & 5.08 & 8.31 & 33.42 & $6.78 \pm 2.78$ & 9.17 & 2.14 & 1.53 & $<32.54$ & 1 \\
\hline $\mathrm{J} 1946+3417$ & $\mathrm{X}$ & $\mathrm{O}$ & 3.17 & 27.02 & 33.59 & $6.97 \pm 2.86$ & 16.10 & 1.01 & 2.96 & $<32.38$ & 3 \\
\hline $\mathrm{J} 2145-0750$ & $\mathrm{Sw}$ & $\mathrm{O}$ & 16.05 & 6.84 & 32.40 & $0.53 \pm 0.22$ & 9.69 & 6.57 & 0.14 & $<29.72$ & 1 \\
\hline $\mathrm{J} 2229+2643$ & $\mathrm{Sw}$ & $\mathrm{O}$ & 2.98 & 93.02 & 33.19 & $1.80 \pm 0.74$ & 45.30 & 0.56 & 1.94 & $<30.95$ & 1 \\
\hline $\mathrm{J} 2317+1439$ & $\mathrm{Sw}$ & $\mathrm{O}$ & 3.45 & 2.46 & 33.34 & $1.43 \pm 0.59$ & 24.20 & 0.89 & 1.99 & $<30.75$ & 1 \\
\hline
\end{tabular}

Note-Inst: C, X, and Sw stand for Chandra, XMM-Newton, and Swift respectively. — Class: I, BW, RB and O stand for isolated MSPs, black-widows, redbacks and others respectively. - References: (1) this work, (2) Espinoza et al. 2013, (3) Prinz et al. 2015 
Table 3. Summary of non-parametric correlation analysis between $L_{x}$ and various pulsar parameters with the censored data.

\begin{tabular}{ccc}
\hline \hline & Kendall's $\tau$ & $p$-value \\
\hline$\dot{E}$ & 0.27 & $2.4 \times 10^{-4}$ \\
$B_{L C}$ & 0.26 & $4.2 \times 10^{-4}$ \\
$B_{S}$ & $-3.1 \times 10^{-3}$ & 0.97 \\
$\tau$ & -0.21 & $4.6 \times 10^{-3}$ \\
\hline \hline
\end{tabular}

Table 4. Summary of the significances ( $p$-value) of K-S and A-D tests.

\begin{tabular}{c|cc|cc|cc|cc|cc|cc|cc|c}
\hline \hline & \multicolumn{2}{|c|}{$B_{\mathrm{S}}$} & \multicolumn{2}{|c|}{$B_{\mathrm{LC}}$} & \multicolumn{2}{|c|}{$\dot{E}$} & \multicolumn{2}{c|}{$\tau$} & \multicolumn{2}{c|}{$L_{x}$} & \multicolumn{3}{c}{$\Gamma$} & \multicolumn{2}{c}{$P_{b}$} \\
& $\mathrm{KS}$ & $\mathrm{AD}$ & $\mathrm{KS}$ & $\mathrm{AD}$ & $\mathrm{KS}$ & $\mathrm{AD}$ & $\mathrm{KS}$ & $\mathrm{AD}$ & $\mathrm{KS}$ & $\mathrm{AD}$ & $\mathrm{KS}$ & $\mathrm{AD}$ & $\mathrm{KS}$ & $\mathrm{AD}$ \\
\hline I vs. O & 0.14 & 0.22 & 0.35 & 0.28 & 0.19 & 0.26 & 0.72 & 0.51 & 0.85 & 0.96 & 0.41 & 0.35 &.. \\
I vs. BWs & 0.38 & 0.18 & 0.080 & 0.028 & 0.080 & 0.046 & 0.080 & 0.15 & 0.19 & 0.27 & 0.67 & 0.49 &.. &.. \\
I vs. RBs & 0.25 & 0.40 & 0.051 & 0.061 & 0.051 & 0.053 & 0.051 & 0.033 & 0.0065 & 0.012 & 0.0036 & 0.0077 &.. &.. \\
O vs. BWs & 0.75 & 0.72 & 0.046 & 0.015 & 0.013 & 0.007 & 0.052 & 0.044 & 0.087 & 0.14 & 0.83 & 0.86 & 0.000003 & 0.00008 \\
O vs. RBs & 0.71 & 0.52 & 0.036 & 0.011 & 0.005 & 0.002 & 0.0052 & 0.0035 & 0.0003 & 0.0009 & 0.0017 & 0.0020 & 0.0001 & 0.0005 \\
BWs vs. RBs & 0.73 & 0.53 & 1.00 & 1.00 & 0.73 & 0.52 & 0.41 & 0.17 & 0.014 & 0.0032 & 0.0074 & 0.010 & 0.081 & 0.10 \\
\hline \hline
\end{tabular}

Note-I, BW, RB and O stand for isolated MSPs, black-widows, redbacks and Others respectively. 


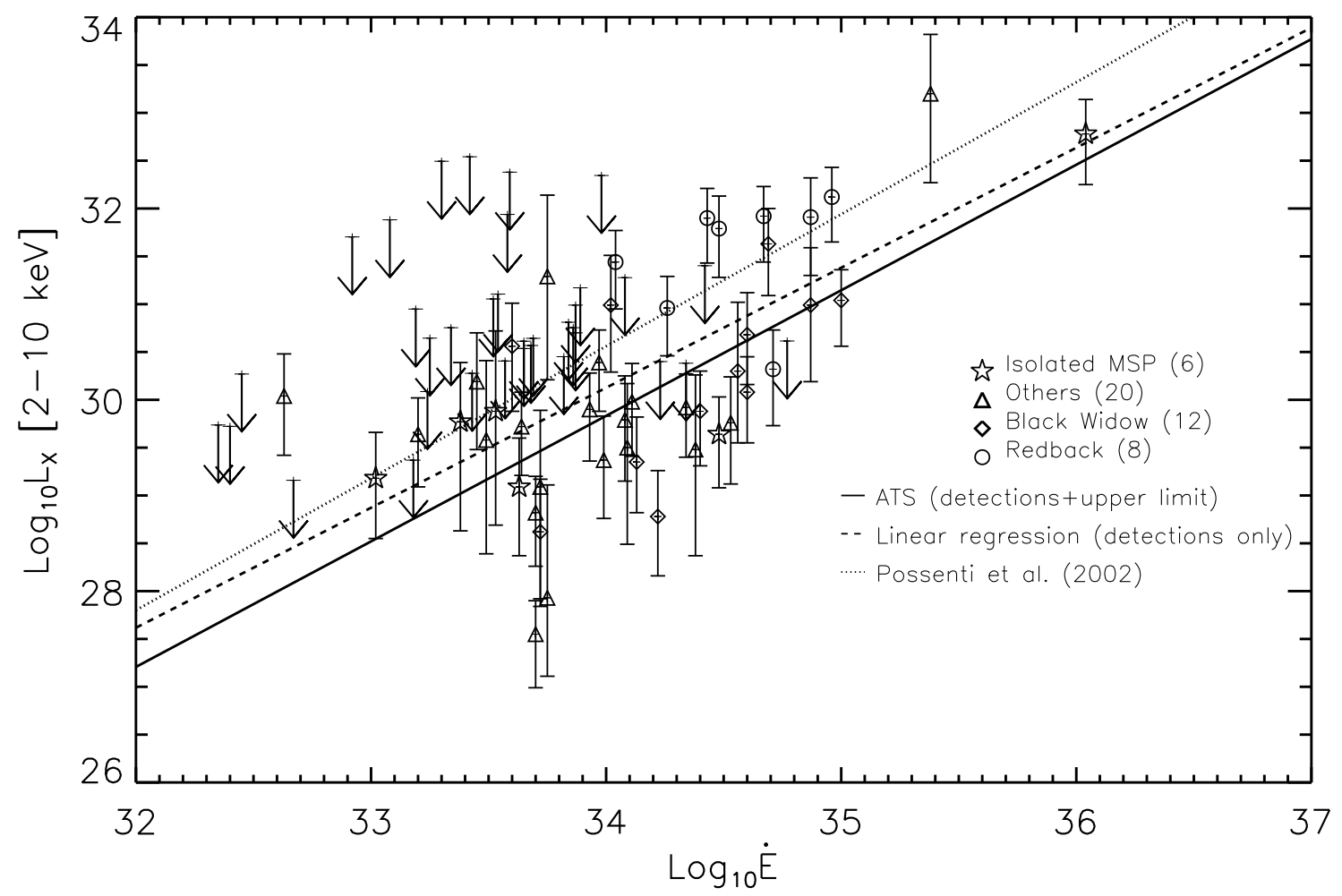

Figure 1. Plot of $L_{x}$ vs $\dot{E}$ for MSPs. Different symbols represent different classes of 46 X-ray detected MSPs with measured $\dot{E}$ (cf. Tab. 1). The arrows illustrate the upper-limits on $L_{x}$ for 35 MSPs with measured $\dot{E}$ given in Tab. 2. The solid line shows the ATS line (Equation 1) as inferred from the censored data. For comparison, we also plotted the relation obtained from the standard linear regression of X-ray detected MSPs only (dashed line) and that reported by Possenti et al. (2002) based on a sample of 10 MSPs (dotted line). 

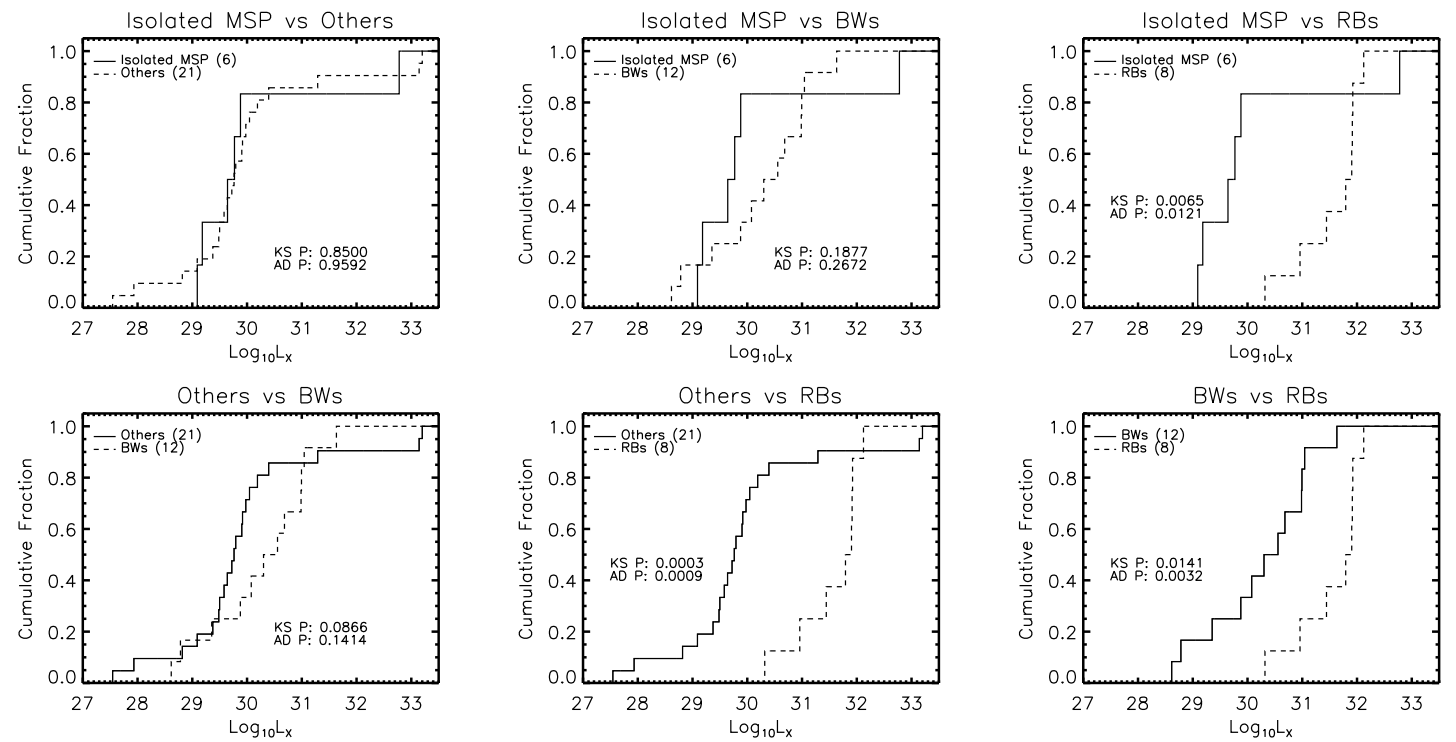

Figure 2. Cumulative distribution functions of $L_{x}$ among different classes of X-ray detected MSPs.
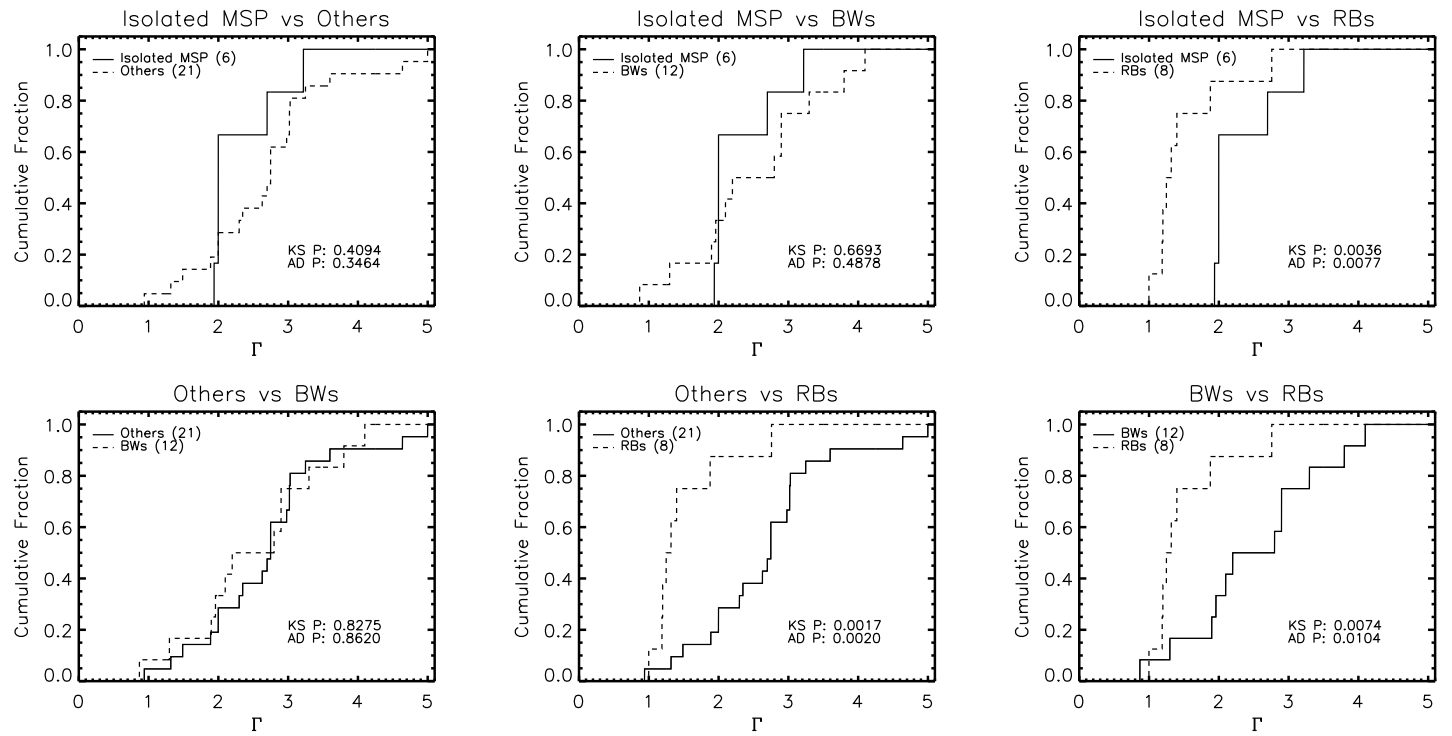

Figure 3. Cumulative distribution functions of $\Gamma$ among different classes of X-ray detected MSPs. 

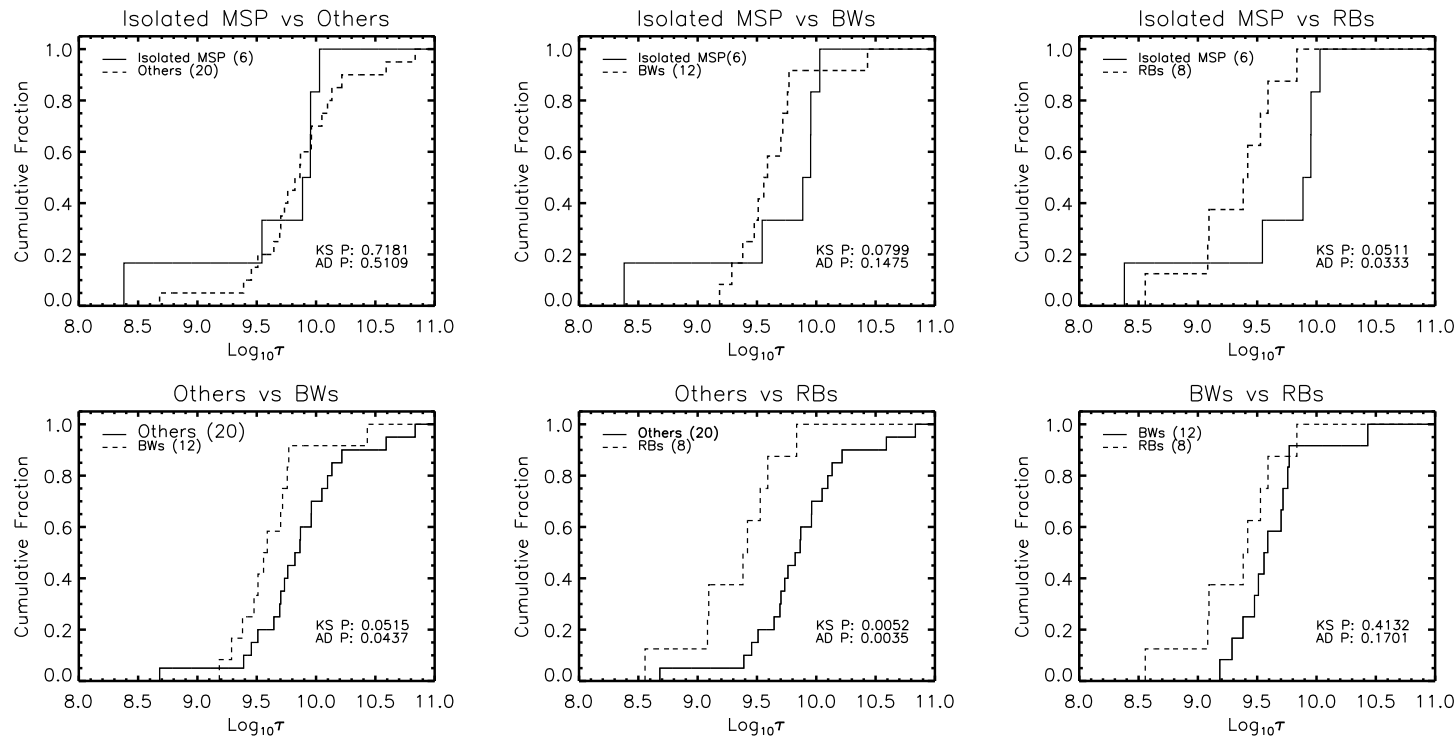

Figure 4. Cumulative distribution functions of $\tau$ among different classes of X-ray detected MSPs.
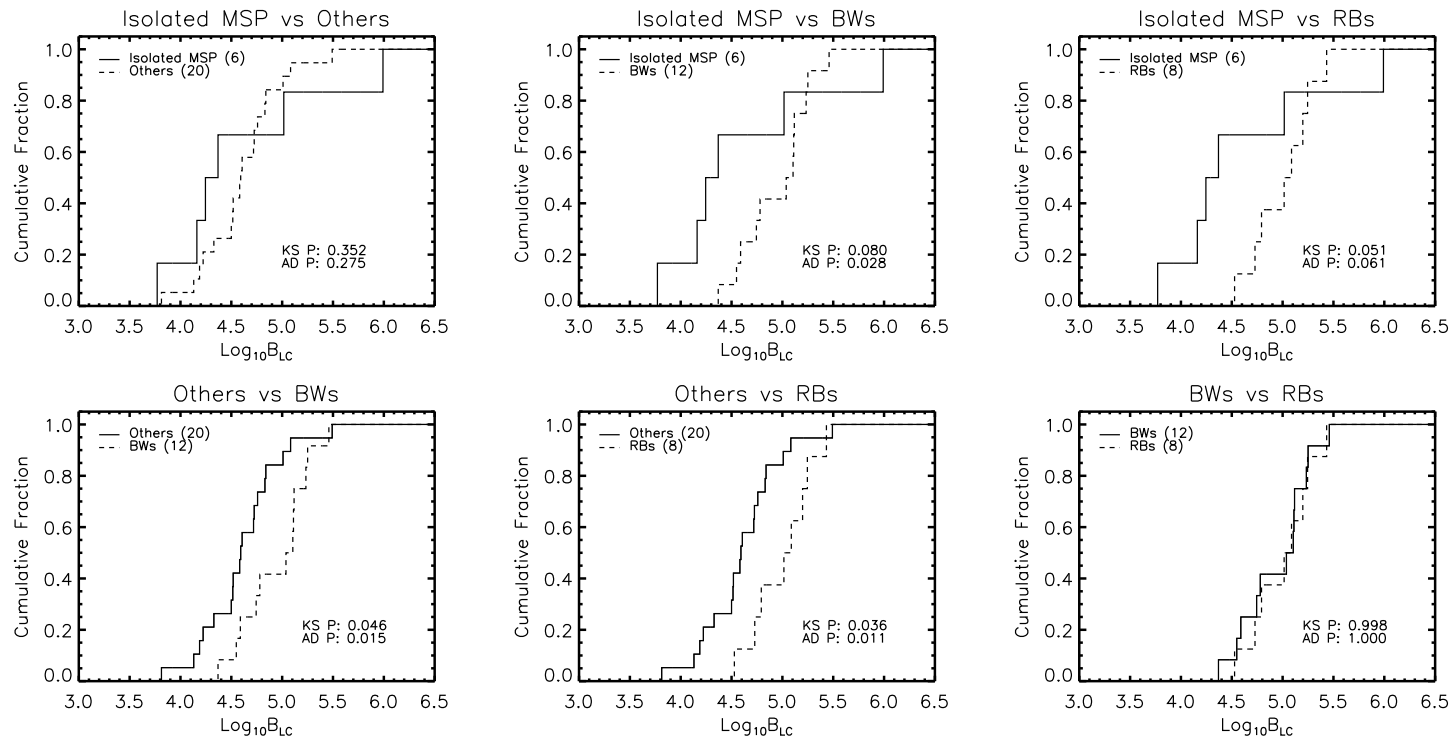

Figure 5. Cumulative distribution functions of $B_{L C}$ among different classes of X-ray detected MSPs. 

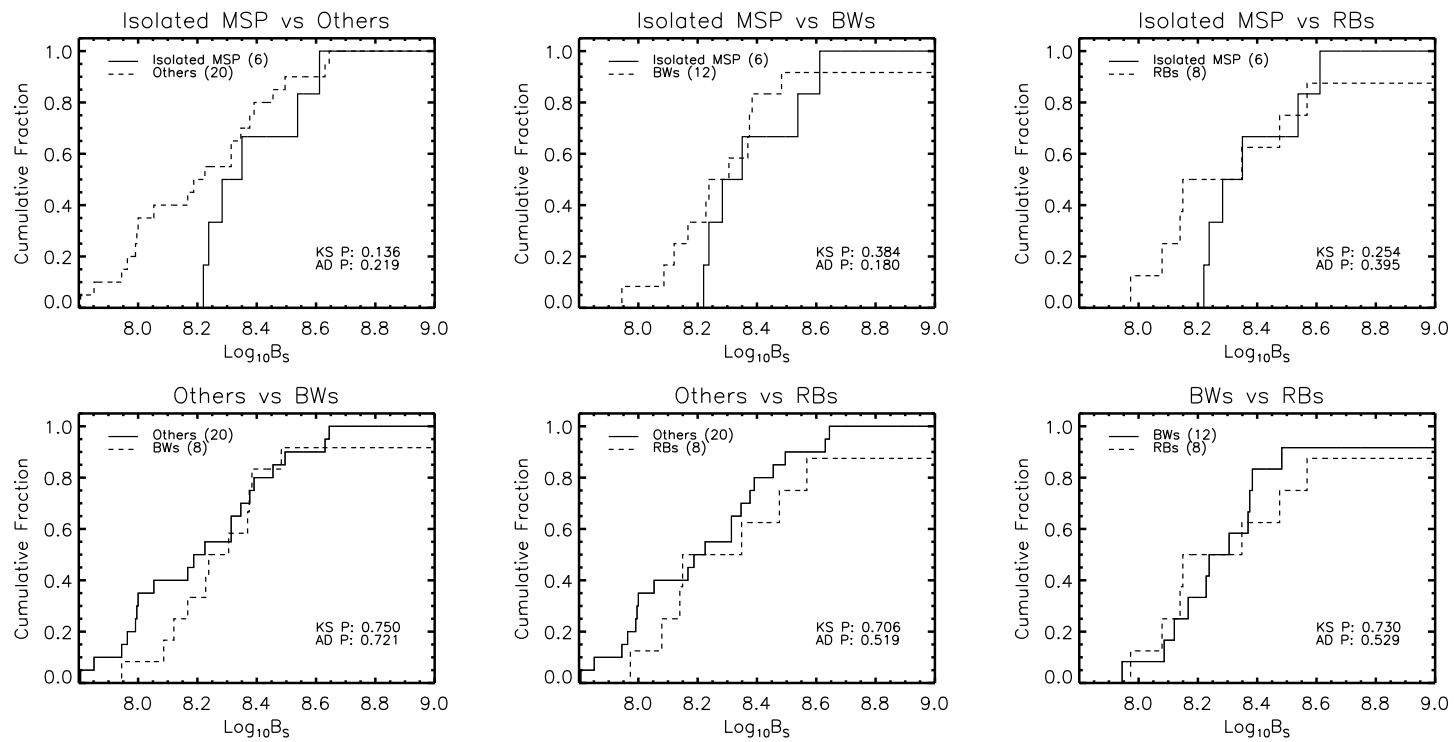

Figure 6. Cumulative distribution functions of $B_{S}$ among different classes of X-ray detected MSPs.
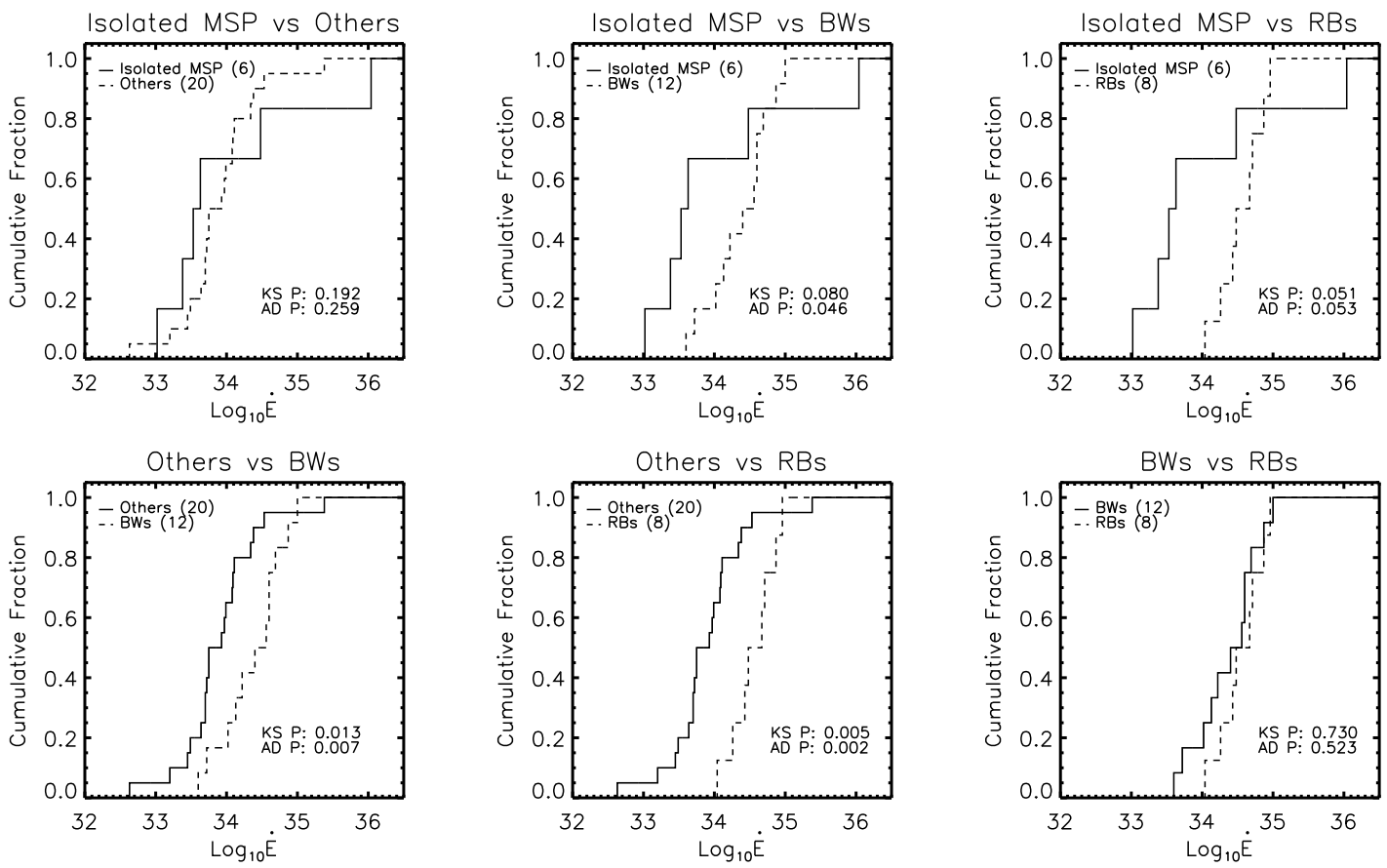

Figure 7. Cumulative distribution functions of $\dot{E}$ among different classes of X-ray detected MSPs. 

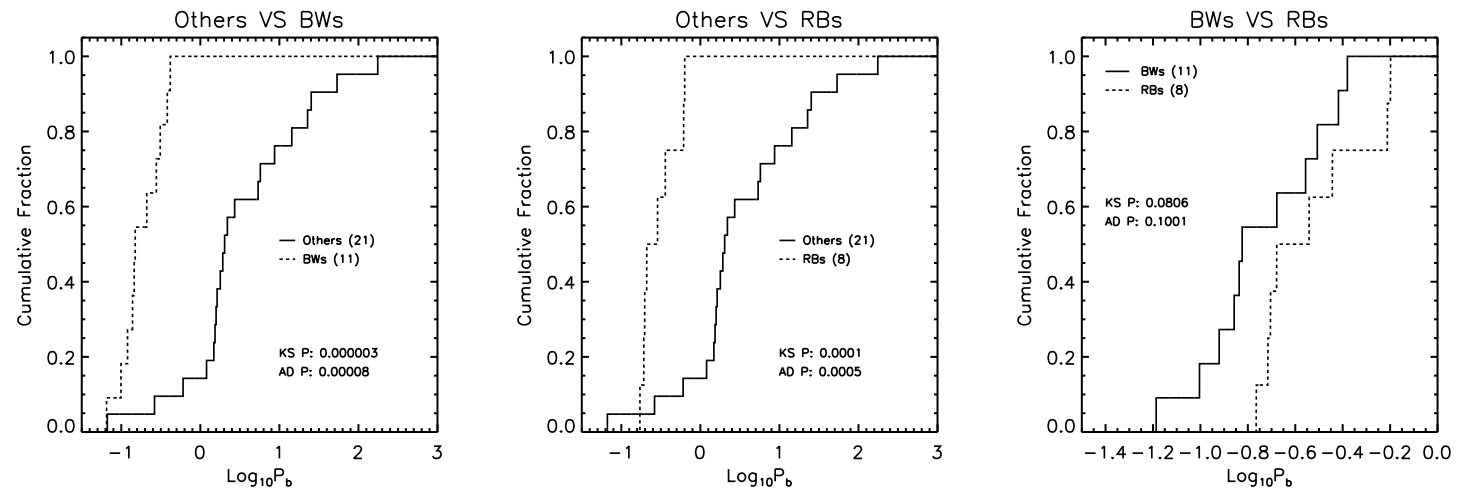

Figure 8. Cumulative distribution functions of $P_{b}$ among different classes of X-ray detected MSPs.
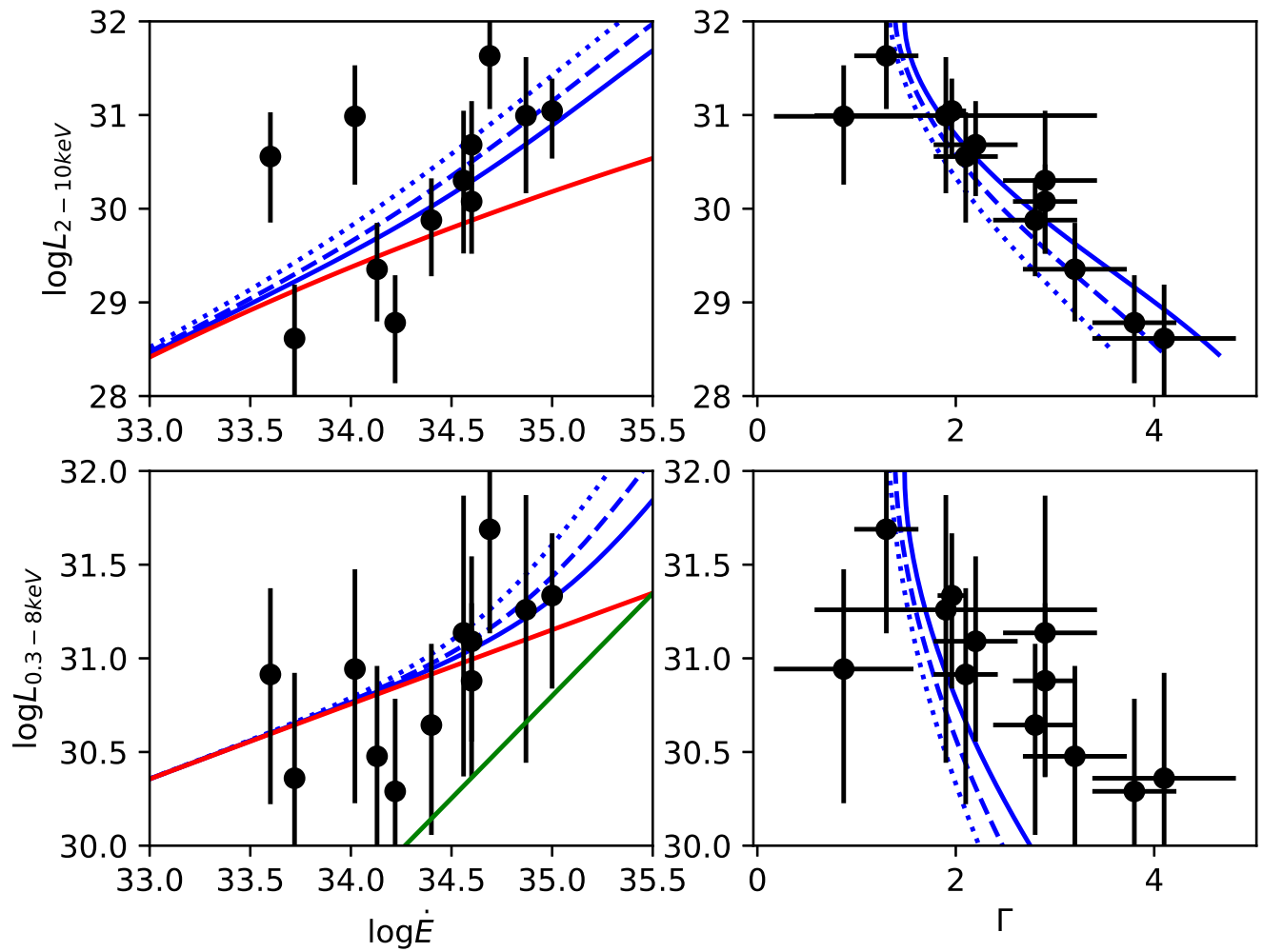

Figure 9. Plots of $L_{x}$ vs $\dot{E}$ (left panels) and vs $\Gamma$ (right panels) for the BW MSPs. The top and bottom show the X-ray properties in $2-10 \mathrm{keV}$ and in $0.3-8 \mathrm{keV}$, respectively. The green and red lines indicates the magnetospheric synchrotron radiation from the secondary pairs [equation (47) in Takata et al. (2012)] and the heated polar cap emission with $B_{s}=3^{8}$ Gauss (see text), respectively. The blue lines are model prediction with the emission from the intra-binary shock and the heated polar cap. The solid, dashed and dotted lines are results for the efficiency $\delta=0.15 \%, 0.3 \%$ and $0.6 \%$, respectively. 

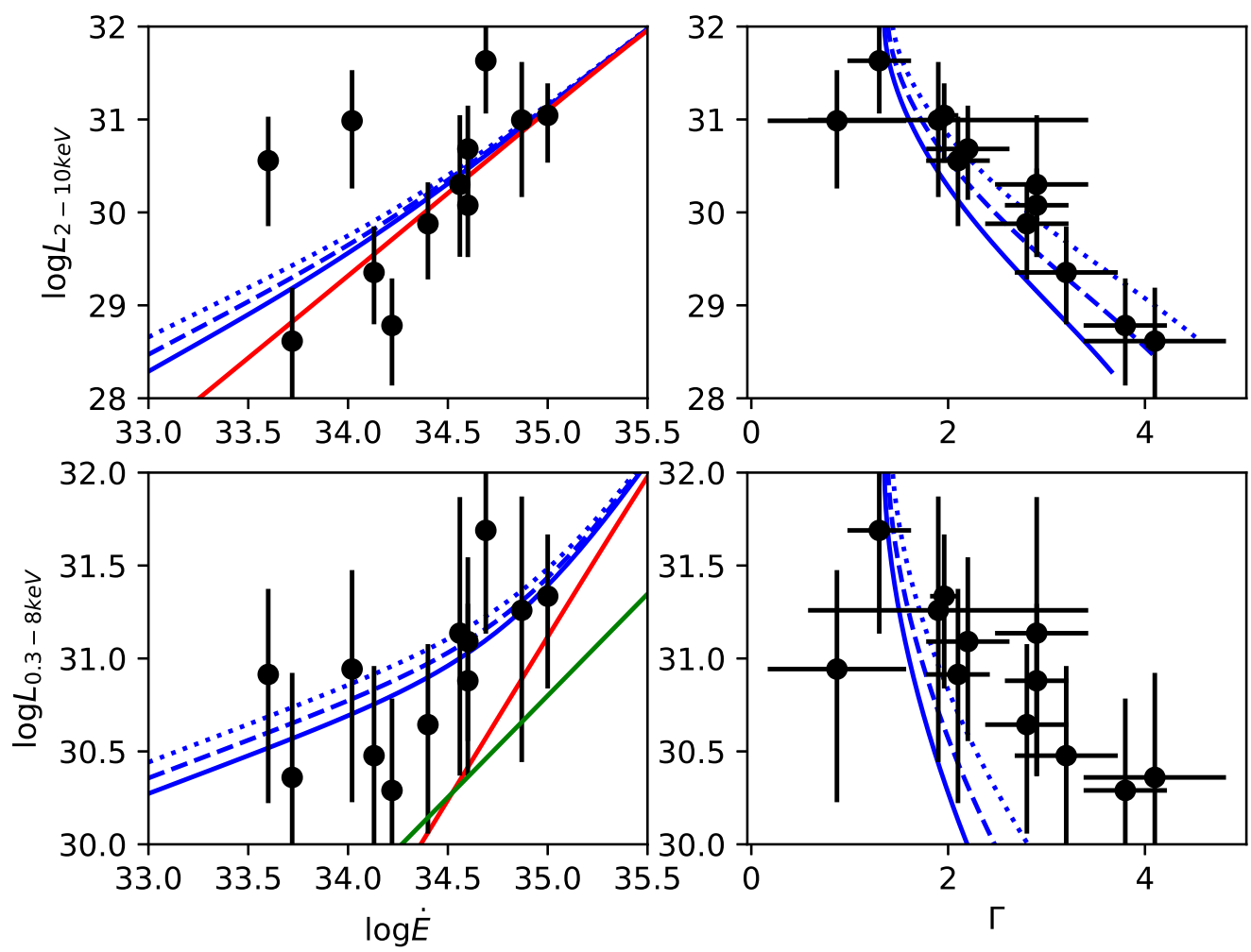

Figure 10. Same as Figure 9, but the different blue color lines are results for the different surface magnetic field strength, which affects the temperature of the heated polar cap, as indicated by equations (4) and (5). The temperatures for the sold, dashed and dotted lines are calculated with $B_{s}=1.5 \times 10^{8} \mathrm{G}, 3 \times 10^{8} \mathrm{G}$ and $6 \times 10^{8} \mathrm{G}$, respectively. The red lines in the top and bottom panels represent the contribution of the shock emission calculated with $\delta=0.3 \%$. 

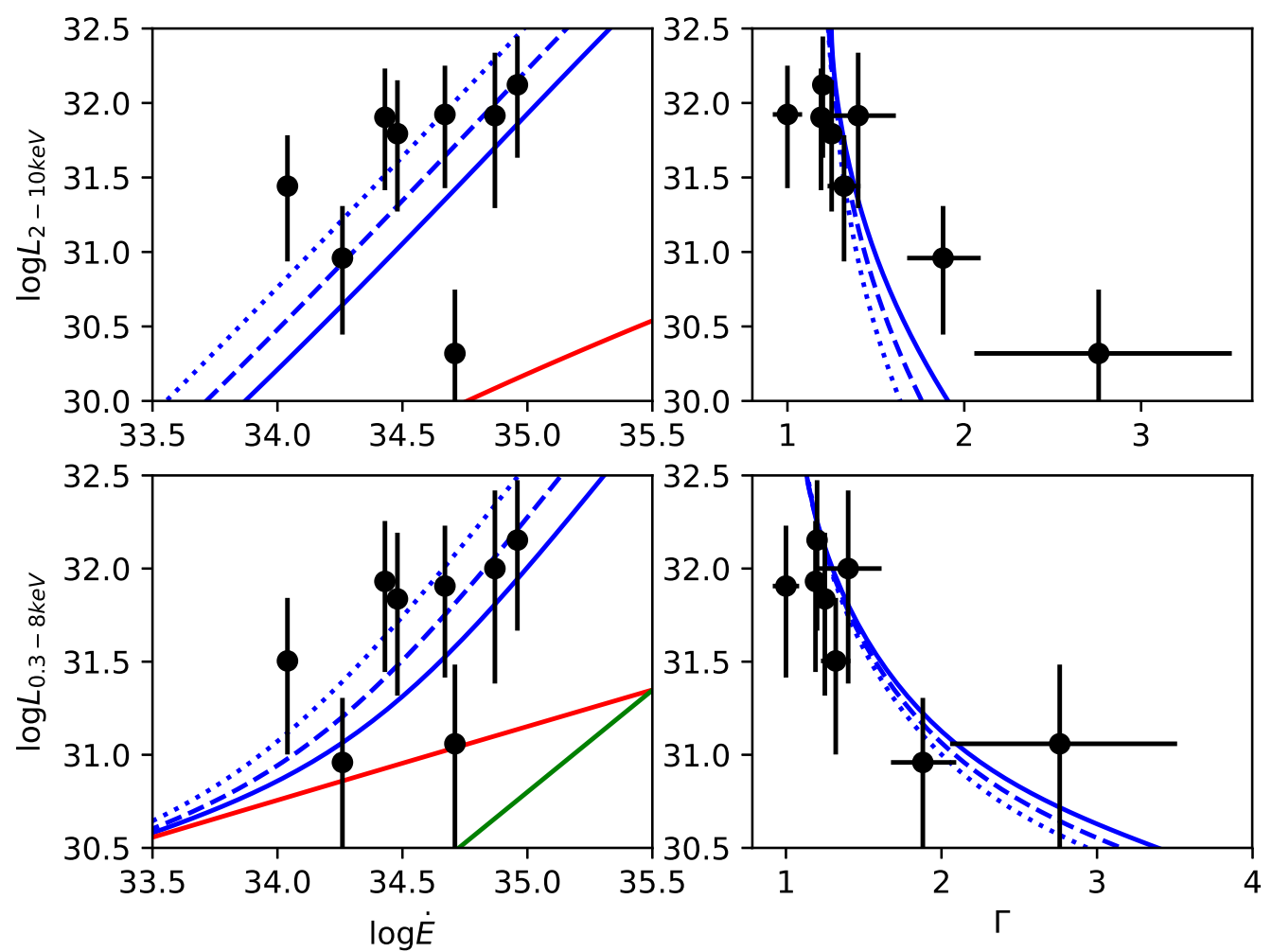

Figure 11. Same as Figure 9, but for the RB MSPs. The solid, dashed and dotted blue lines are results for $\delta=2 \%, 4 \%$ and $8 \%$, respectively. 

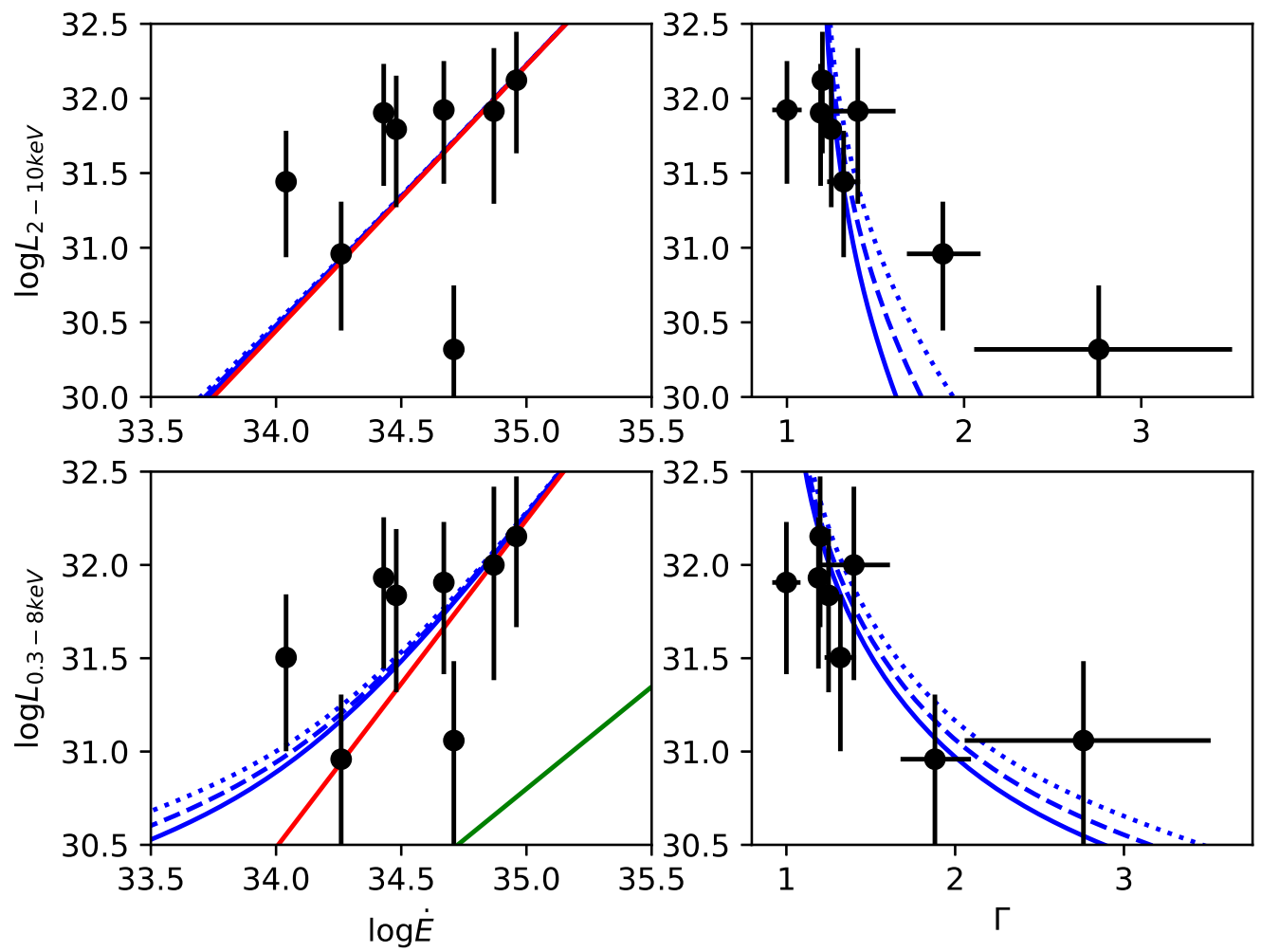

Figure 12. Same as Figure 10, but for the RB MSPs. The contribution of the shocked emission (red lines) is calculated with $\delta=4 \%$. 